\title{
Metalloporphyrin-Catalyzed Diastereoselective Epoxidation of Allyl-Substituted Alkenes
}

\author{
Wing-Kei Chan, Man-Kin Wong, * and Chi-Ming Che* \\ Department of Chemistry and Open Laboratory of Chemical Biology of the Institute of \\ Molecular Technology for Drug Discovery and Synthesis, The University of Hong Kong, \\ Pokfulam Road, Hong Kong, China
}

cmche@hku.hk

\section{SUPPORTING INFORMATION}

\section{Table of Contents}

General Structures of Metalloporphyrin Catalysts

Literature References for Alkenes $\mathbf{3}$ and $\mathbf{5}$

S3-S4

Literature References for Epoxides 4b, 6a, 6c, 6e-6h and 8

S5-S6

Characterization Data of Epoxides $4 \mathbf{a}-\mathbf{4 e}, \mathbf{6 b}$ and $\mathbf{6 d}$

S7-S11

${ }^{1}$ H NMR Spectra of Diastereomeric Mixtures of Epoxides 4 and 6

S12-S14

Determination of the $\boldsymbol{\alpha - 8} / \boldsymbol{\beta}-\mathbf{8}$ ratio with ${ }^{1} \mathrm{H}$ NMR

S15

${ }^{1} \mathrm{H}$ NMR Spectra of $\mathbf{4 a - 4 e , ~} \mathbf{6 b}$ and $\mathbf{6 d}$

S16-S23

${ }^{13} \mathrm{C}$ NMR Spectra $4 \mathbf{a}-\mathbf{4 e}, \mathbf{6 b}$ and $\mathbf{6 d}$

S24-S31 


\section{General Structures of Metalloporphyrin Catalysts}

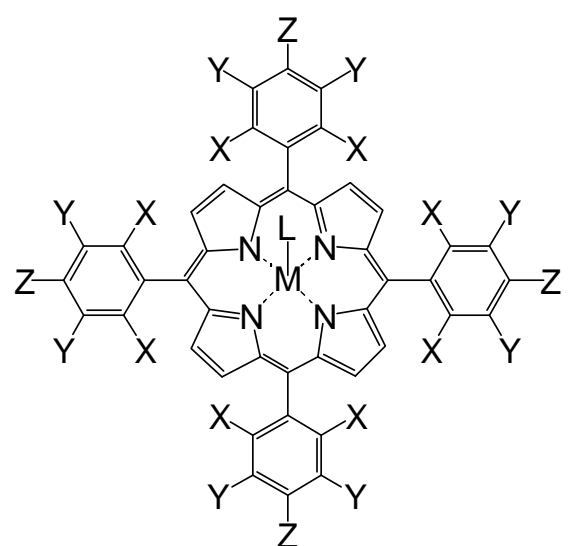

1: $\mathrm{M}=\mathrm{Mn}^{3+} ; \mathrm{X}=\mathrm{Cl} ; \mathrm{Y}=\mathrm{Z}=\mathrm{H} ; \mathrm{L}=\mathrm{Cl} \quad \mathrm{Mn}(\mathrm{TDCPP}) \mathrm{Cl}$

2: $\mathrm{M}=\mathrm{Ru}^{2+} ; \mathrm{X}=\mathrm{Cl} ; \mathrm{Y}=\mathrm{Z}=\mathrm{H} ; \mathrm{L}=\mathrm{CO} \quad \mathrm{Ru}(\mathrm{TDCPP})(\mathrm{CO})$

$\mathrm{M}=\mathrm{Mn}^{3+} ; \mathrm{X}=\mathrm{Z}=\mathrm{CH}_{3} ; \mathrm{Y}=\mathrm{H} ; \mathrm{L}=\mathrm{Cl} \quad \mathrm{Mn}(\mathrm{TMP}) \mathrm{Cl}$

$\mathrm{M}=\mathrm{Mn}^{3+} ; \mathrm{X}=\mathrm{Y}=\mathrm{Z}=\mathrm{F} ; \mathrm{L}=\mathrm{Cl} \quad \mathrm{Mn}\left(\mathrm{F}_{20^{-}} \mathrm{TPP}\right) \mathrm{Cl}$

$\mathrm{M}=\mathrm{Mn}^{3+} ; \mathrm{X}=\mathrm{Y}=\mathrm{H} ; \mathrm{Z}=\mathrm{CH}_{3} ; \mathrm{L}=\mathrm{Cl} \quad \mathrm{Mn}(\mathrm{TTP}) \mathrm{Cl}$ 


\section{Literature References for Alkenes 3a-3e and 5a-5h}

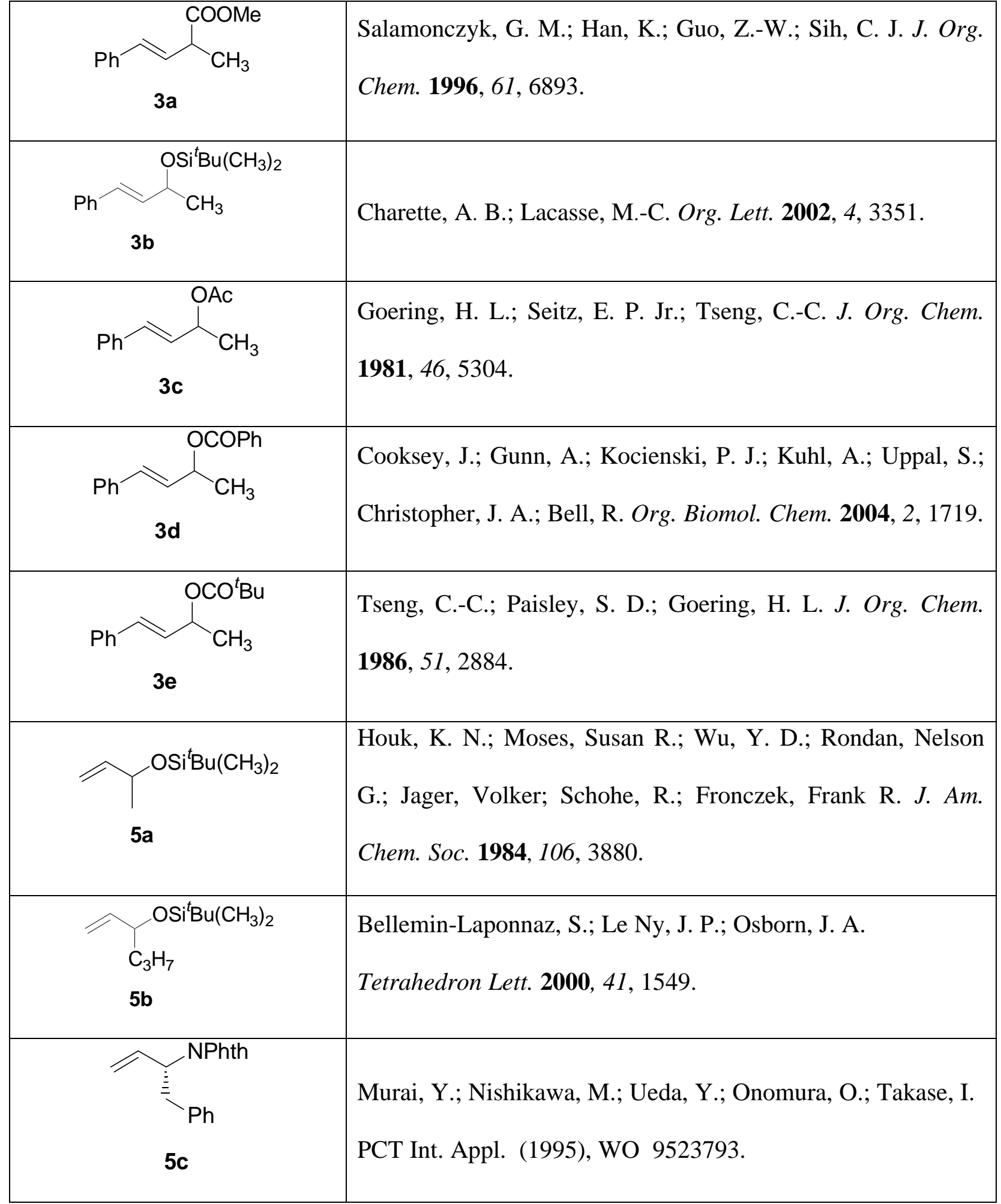




\begin{tabular}{|l|l|}
\hline & Skowronek, P.; Gawronski, J. Tetrahedron Lett. 2000, 41, \\
\hline Luly, J. R.; Dellaria, J. F.; Plattner, J. J.; Soderquist, J. L.; Yi, \\
\hline N. J. Org. Chem. 1987, 52, 1487.
\end{tabular}


Literature References for Epoxides 4b, 6a, 6c, 6e-6h and 8

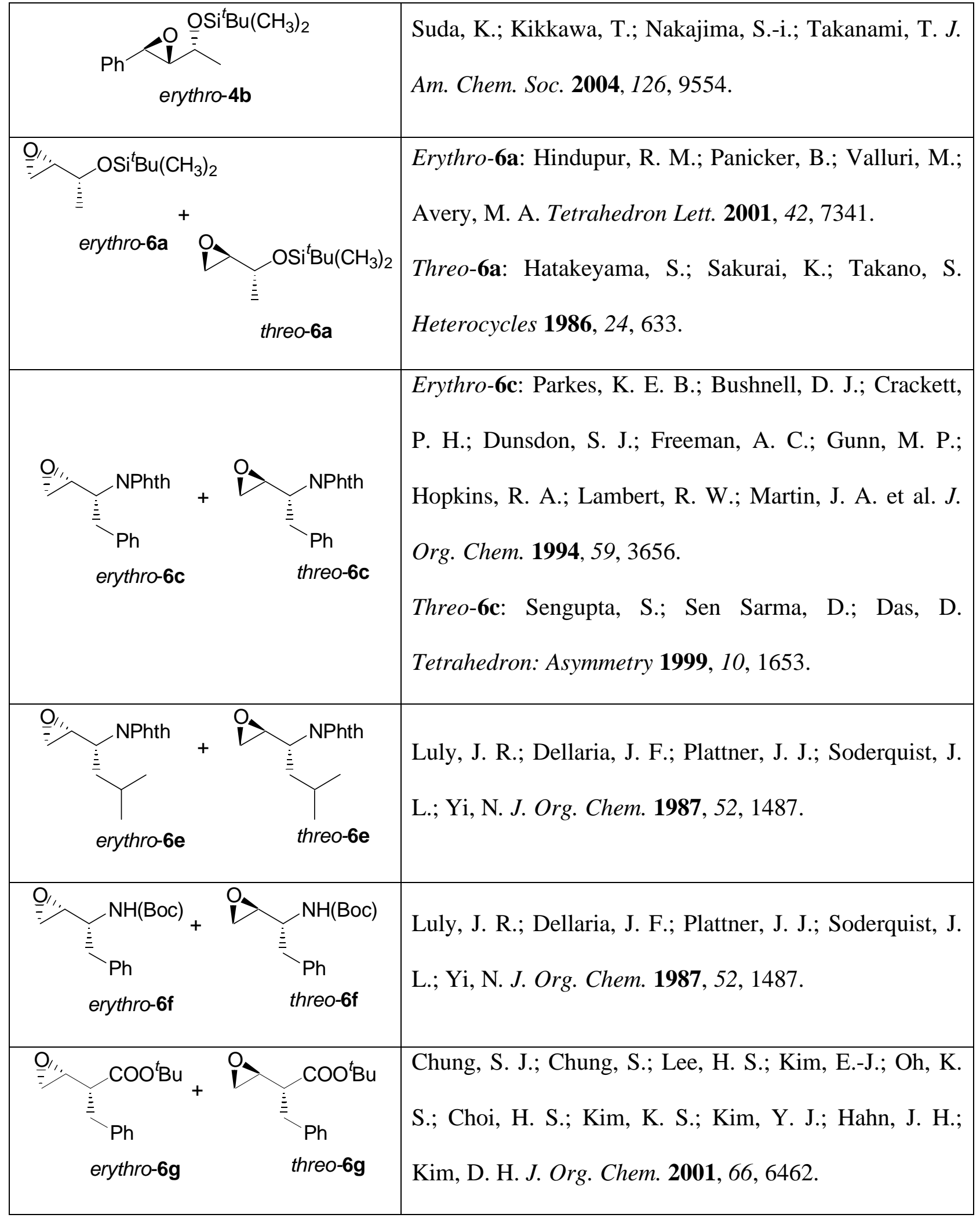




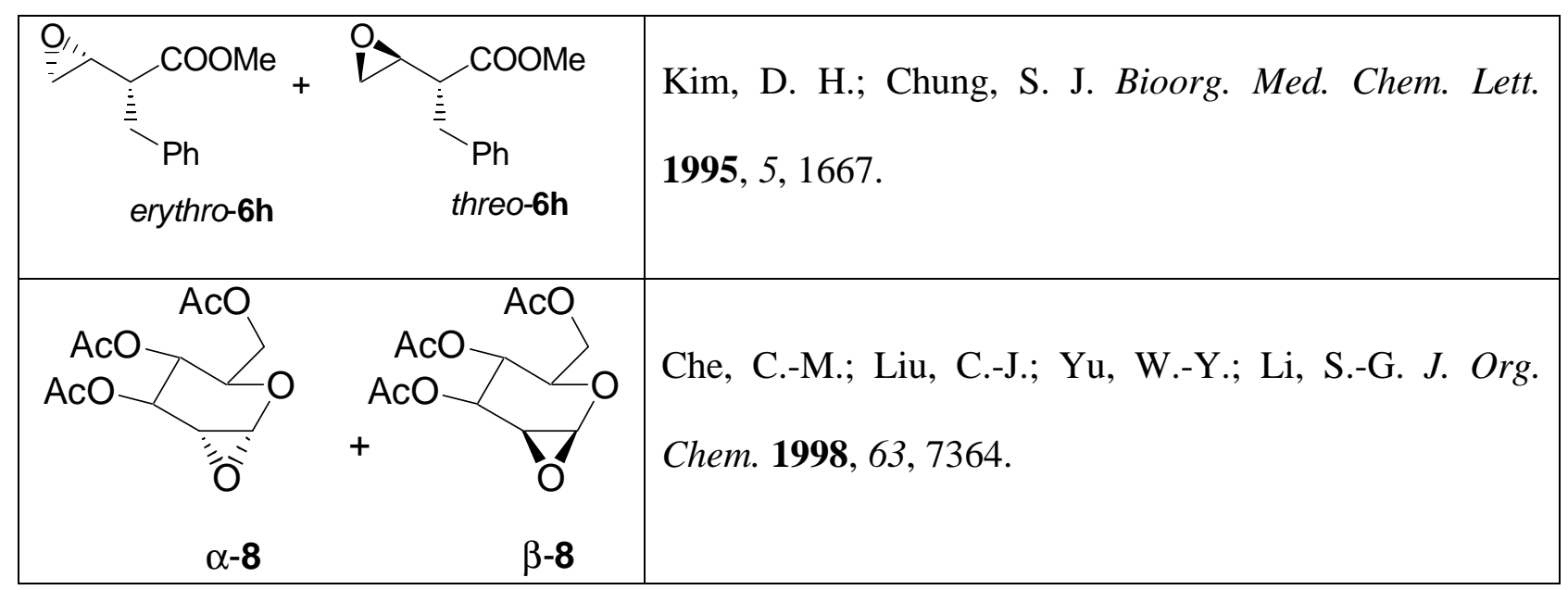




\section{Characterization Data of Epoxides $4 a-4 e, 6 b$ and $6 d$}<smiles>COC(=O)C(C)C1OC1c1ccccc1</smiles>

erythro-4a

2

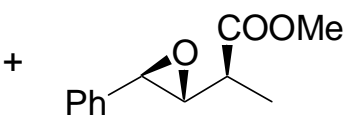

threo-4a

1

Colorless oil, analytical TLC (silica gel 60) (10\% EA in hexane), erythro-4a $\mathrm{R}_{f}=0.26$, threo-4a $\mathrm{R}_{f}=0.3 ;{ }^{1} \mathrm{H}$ NMR $\left(400 \mathrm{MHz}, \mathrm{CDCl}_{3}\right)$ ä 7.35-7.26 (m, 5H), $3.8(\mathrm{~d}, J=2.0 \mathrm{~Hz}$, threo- $1 / 3 \times 1 \mathrm{H}), 3.76(\mathrm{~s}$, erythro- $2 / 3 \times 3 \mathrm{H}), 3.71(\mathrm{~s}$, threo- $1 / 3 \times 1 \mathrm{H}), 3.70(\mathrm{~d}, J=2.0$ $\mathrm{Hz}$, erythro- $1 / 3 \times 1 \mathrm{H}), 3.22(\mathrm{dd}, \mathrm{J}=7.3,2.0 \mathrm{~Hz}$, erythro- $2 / 3 \times 3 \mathrm{H}), 3.12(\mathrm{dd}, J=7.1$, $2.0 \mathrm{~Hz}$, threo- $1 / 3 \times 1 \mathrm{H}), 2.58-2.51(\mathrm{~m}, 1 \mathrm{H}), 1.37(\mathrm{~d}, J=7.1 \mathrm{~Hz}$, threo- $1 / 3 \times 3 \mathrm{H}), 1.29$ $(\mathrm{d}, J=7.3 \mathrm{~Hz}$, erythro- $2 / 3 \times 3 \mathrm{H}) ;{ }^{13} \mathrm{C} \mathrm{NMR}\left(100.61 \mathrm{MHz}, \mathrm{CDCl}_{3}\right)$ ä 174.0, 136.8, $128.54,128.50,128.3,128.2,125.6,63.1,62.9,58.1,57.2,52.1,52.0,42.5,13.7,13.2$; IR (KBr) $1735 \mathrm{~cm}^{-1}$; EIMS m/z $206\left(\mathrm{M}^{+}\right)$; HRMS (EI) for $\mathrm{C}_{12} \mathrm{H}_{14} \mathrm{O}_{3}$, calcd 206.0942, found 206.0937 .

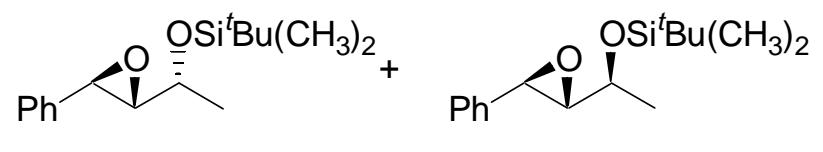

erythro-4b

1 threo-4b

1

Colorless oil, analytical TLC (silica gel 60) (2\% EA in hexane), erythro-4b $\mathbf{R}_{f}=0.35$, threo-4b $\mathrm{R}_{f}=0.35 ;{ }^{1} \mathrm{H} \mathrm{NMR}\left(400 \mathrm{MHz}, \mathrm{CDCl}_{3}\right)$ ä 7.35-7.24 (m, 5H), 3.92-3.86 (m, erythro- $1 / 2 \times 1 \mathrm{H}), 3.82(\mathrm{~d}, J=1.9 \mathrm{~Hz}$, erythro- $1 / 2 \times 1 \mathrm{H}), 3.78-3.72(\mathrm{~m}$, threo- $1 / 2 \times$ $1 \mathrm{H}), 3.73(\mathrm{~d}, \mathrm{~J}=2.1 \mathrm{~Hz}$, threo- $1 / 2 \times 1 \mathrm{H}), 3.00(\mathrm{dd}, J=5.7,2.0 \mathrm{~Hz}$, threo- $1 / 2 \times 1 \mathrm{H})$, $2.91(\mathrm{dd}, J=4.2,2.1 \mathrm{~Hz}$, erythro- $1 / 2 \times 1 \mathrm{H}), 1.28(\mathrm{~d}, J=6.4 \mathrm{~Hz}$, erythro- $1 / 2 \times 3 \mathrm{H}), 1.26$ $(\mathrm{d}, J=6.5 \mathrm{~Hz}$, threo- $1 / 2 \times 3 \mathrm{H}), 0.93(\mathrm{~s}$, threo- $1 / 2 \times 9 \mathrm{H}), 0.89$ (s, erythro- $1 / 2 \times 9 \mathrm{H})$, 
$0.15(\mathrm{~s}, 1 / 2 \times 3 \mathrm{H}), 0.11(\mathrm{~s}, 1 / 2 \times 3 \mathrm{H}), 0.10(\mathrm{~s}, 1 / 2 \times 3 \mathrm{H}), 0.09(\mathrm{~s}, 1 / 2 \times 3 \mathrm{H}) ;{ }^{13} \mathrm{C} \mathrm{NMR}$ $\left(100.61 \mathrm{MHz}, \mathrm{CDCl}_{3}\right)$ ä 137.5, 137.2, 128.4, 128.3, 128.0, 127.9, 125.6, 125.5, 69.3, $67.4,66.7,66.0,56.1,56.0,25.8,25.7,20.8,20.3,18.19,18.16,-4.61,-4.65,-4.8 ;$ IR (KBr) 2956, 1463, $836 \mathrm{~cm}^{-1}$ EIMS m/z, $221\left(\mathrm{M}^{+}-\mathrm{C}_{4} \mathrm{H}_{9}\right)$; HRMS (EI) for $\mathrm{C}_{12} \mathrm{H}_{17} \mathrm{O}_{2} \mathrm{Si}\left(\mathrm{M}^{+}\right.$ - $\left.\mathrm{C}_{4} \mathrm{H}_{9}\right)$, calcd 221.0998, found 221.0996.

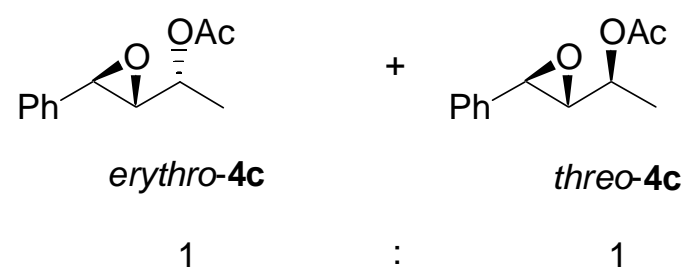

Colorless oil, analytical TLC (silica gel 60) (20\% EA in hexane), erythro-4c $\mathrm{R}_{f}=0.38$, threo-4c $\mathrm{R}_{f}=0.44 ;{ }^{1} \mathrm{H}$ NMR (400 MHz, $\left.\mathrm{CDCl}_{3}\right)$ ä 7.34-7.25 (m, 5H), 4.98-4.91 (m, 1H), $3.86(\mathrm{~d}, J=2.0 \mathrm{~Hz}$, threo- $1 / 2 \times 1 \mathrm{H}), 3.78(\mathrm{~d}, J=2.0 \mathrm{~Hz}$, erythro- $1 / 2 \times 1 \mathrm{H}), 3.13(\mathrm{dd}, J=$ 5.8, $2.0 \mathrm{~Hz}$, erythro- $1 / 2 \times 1 \mathrm{H}), 3.03(\mathrm{dd}, \mathrm{J}=4.8,2.0 \mathrm{~Hz}$, threo- $1 / 2 \times 1 \mathrm{H}), 2.09(\mathrm{~s}$, erythro- $1 / 2 \times 3 \mathrm{H}), 2.05(\mathrm{~s}$, threo- $1 / 2 \times 3 \mathrm{H}), 1.35(\mathrm{~d}, J=6.6 \mathrm{~Hz}, 3 \mathrm{H}) ;{ }^{13} \mathrm{C}$ NMR $(100.62$ $\left.\mathrm{MHz}, \mathrm{CDCl}_{3}\right)$ ä $170.2,136.7,136.4,128.55,128.49,128.42,128.3,125.66,125.61,70.3$, 69.2, 63.4, 62.9, 56.9, 56.1, 21.1, 21.0, 16.5, 16.2; IR (KBr) $1720 \mathrm{~cm}^{-1}$; EIMS m/z 206 $\left(\mathrm{M}^{+}\right)$; HRMS (EI) for $\mathrm{C}_{12} \mathrm{H}_{14} \mathrm{O}_{3}$, calcd 206.0942, found 206.0951 . 


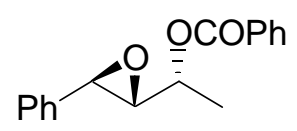

erythro-4d

3

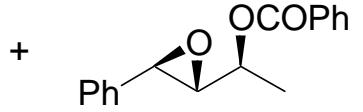

threo-4d

2

Colorless oil, analytical TLC (silica gel 60) (20\% EA in hexane) erythro-4d $\mathrm{R}_{f}=0.48$, threo-4d $\mathrm{R}_{f}=0.53 ;{ }^{1} \mathrm{H}$ NMR (400 $\left.\mathrm{MHz}, \mathrm{CDCl}_{3}\right)$ ä 8.10-8.08 (m, 2H), 7.56-7.27 (m, $8 \mathrm{H}), 5.24-5.20(\mathrm{~m}, 1 \mathrm{H}), 3.96(\mathrm{~d}, J=2.0 \mathrm{~Hz}$, threo- $2 / 5 \times 1 \mathrm{H}), 3.86(\mathrm{~d}, J=2.0 \mathrm{~Hz}$, erythro- $3 / 5 \times 1 \mathrm{H}), 3.27(\mathrm{dd}, J=5.5,2.0 \mathrm{~Hz}$, erythro- $3 / 5 \times 1 \mathrm{H}), 3.16(\mathrm{dd}, J=4.8,2.0 \mathrm{~Hz}$, threo- $2 / 5 \times 1 \mathrm{H}), 1.49(\mathrm{~d}, J=6.5 \mathrm{~Hz}$, erythro- $3 / 5 \times 1 \mathrm{H}), 1.48(\mathrm{~d}, J=6.6 \mathrm{~Hz}$, threo- $2 / 5 \mathrm{x}$ $1 \mathrm{H}) ;{ }^{13} \mathrm{C}$ NMR $\left(100.62 \mathrm{MHz}, \mathrm{CDCl}_{3}\right)$ ä 165.66, 165.64, 136.5, 136.3, 133.1, 129.78, $129.74,128.6,128.5,128.48,128.45,128.3,125.7,125.6,70.5,69.8,63.5,63.0,57.0$, 56.0, 16.6, 16.2; IR (KBr) $1709 \mathrm{~cm}^{-1}$; EIMS $\mathrm{m} / z 268\left(\mathrm{M}^{+}\right)$; HRMS (EI) for $\mathrm{C}_{17} \mathrm{H}_{16} \mathrm{O}_{3}$, calcd 268.1099, found 268.1090.

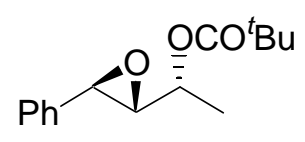

erythro-4e

Colorless oil, analytical TLC (silica gel 60) (10\% EA in hexane), erythro-4e $\mathrm{R}_{f}=0.44$; ${ }^{1} \mathrm{H}$ NMR $\left(300 \mathrm{MHz}, \mathrm{CDCl}_{3}\right)$ ä 7.36-7.25 (m, 5H), 4.97-4.89 (m, 1H), $3.78(\mathrm{~d}, J=2.0$ $\mathrm{Hz}, 1 \mathrm{H}), 3.15(\mathrm{dd}, J=5.7,2.1 \mathrm{~Hz}, 1 \mathrm{H}), 1.34(\mathrm{~d}, J=6.5 \mathrm{~Hz}, 3 \mathrm{H}), 1.24(\mathrm{~s}, 9 \mathrm{H}) ;{ }^{13} \mathrm{C} \mathrm{NMR}$ $\left(100.62 \mathrm{MHz}, \mathrm{CDCl}_{3}\right)$ ä 177.7, 136.5, 128.5, 128.3, 125.6, 69.7, 63.5, 56.0, 38.8, 27.1, 16.4; IR (KBr) $1730 \mathrm{~cm}^{-1}$; EIMS $\mathrm{m} / z 248\left(\mathrm{M}^{+}\right)$; HRMS (EI) for $\mathrm{C}_{15} \mathrm{H}_{20} \mathrm{O}_{3}$, calcd 248.1412, found 248.1411. 


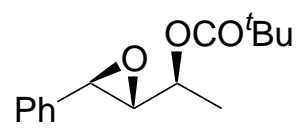

threo-4e

Colorless oil, analytical TLC (silica gel 60) (10\% EA in hexane), threo-4e $\mathrm{R}_{f}=0.49 ;{ }^{1} \mathrm{H}$ NMR (300 MHz, $\left.\mathrm{CDCl}_{3}\right)$ ä 7.35-7.25 (m, 5H), 5.01-4.97 (m, 1H), $3.85(\mathrm{~d}, J=2.0 \mathrm{~Hz}$, $1 \mathrm{H}), 3.01(\mathrm{dd}, J=5.6,2.0 \mathrm{~Hz}, 1 \mathrm{H}), 1.35(\mathrm{~d}, J=6.5 \mathrm{~Hz}, 3 \mathrm{H}), 1.21(\mathrm{~s}, 9 \mathrm{H}) ;{ }^{13} \mathrm{C} \mathrm{NMR}$ $\left(75.47 \mathrm{MHz}, \mathrm{CDCl}_{3}\right)$ ä 177.7, 136.8, 128.4, 128.2, 125.5, 68.7, 63.1, 56.5, 38.8, 27.1, 16.3; IR (KBr) $1731 \mathrm{~cm}^{-1}$; EIMS $\mathrm{m} / z 248\left(\mathrm{M}^{+}\right)$; HRMS (EI) for $\mathrm{C}_{15} \mathrm{H}_{20} \mathrm{O}_{3}$, calcd 248.1412 , found 248.1413 .

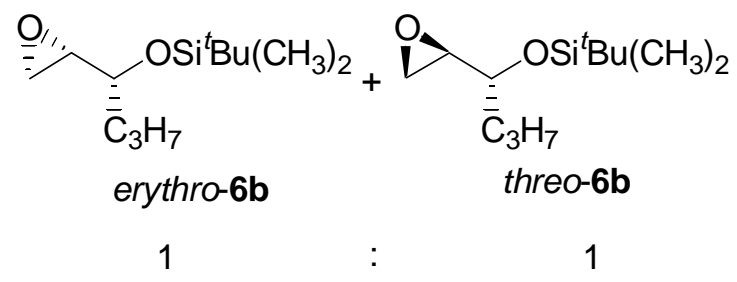

Colorless oil; analytical TLC (silica gel 60) (5\% EA in hexane), erythro-6b $\mathrm{R}_{f}=0.51$, threo-6b $\mathrm{R}_{f}=0.42 ;{ }^{1} \mathrm{H}$ NMR $\left(300 \mathrm{MHz}, \mathrm{CDCl}_{3}\right)$ ä $3.56(\mathrm{dd}, J=10.9,4.9 \mathrm{~Hz}$, erythro- $1 / 2$ x $1 \mathrm{H}), 3.29-3.23(\mathrm{~m}$, threo- $1 / 2 \times 1 \mathrm{H}), 2.93-2.88(\mathrm{~m}$, threo- $1 / 2 \times 1 \mathrm{H}), 2.87-2.84(\mathrm{~m}$, erythro- $1 / 2 \times 1 \mathrm{H}), 2.78-2.75(\mathrm{~m}$, threo- $1 / 2 \times 1 \mathrm{H}), 2.69(\mathrm{dd}, J=5.4,3.9 \mathrm{~Hz}$, erythro- $1 / 2$ x $1 \mathrm{H}), 2.64(\mathrm{dd}, J=5.5,2.7 \mathrm{~Hz}$, erythro- $1 / 2 \times 1 \mathrm{H}), 2.54(\mathrm{dd}, J=4.9,2.7 \mathrm{~Hz}$, threo- $1 / 2 \mathrm{x}$ $1 \mathrm{H}), 1.56-1.32(\mathrm{~m}, 4 \mathrm{H}), 0.95-0.87(\mathrm{~m}, 3 \mathrm{H}), 0.90(\mathrm{~s}$, threo- $1 / 2 \times 9 \mathrm{H}), 0.88$ (s, erythro1/2 x 9H), 0.10-0.04 (m, 6H); ${ }^{13} \mathrm{C}$ NMR (75.47 MHz, $\left.\mathrm{CDCl}_{3}\right)$ ä 74.3, 71.0, 55.9, 54.6, $44.76,44.72,37.4,36.8,25.77,25.72,18.4,18.0,14.1,14.0,-4.4,-4.9,-5.1$; IR (KBr) 2958, 1463, $836 \mathrm{~cm}^{-1}$; EIMS m/z $215\left(\mathrm{M}^{+}-\mathrm{CH}_{3}\right)$; HRMS (EI) for $\mathrm{C}_{11} \mathrm{H}_{23} \mathrm{O}_{2} \mathrm{Si}\left(\mathrm{M}^{+}-\right.$ $\mathrm{CH}_{3}$ ), calcd 215.1467, found 215.1445. 


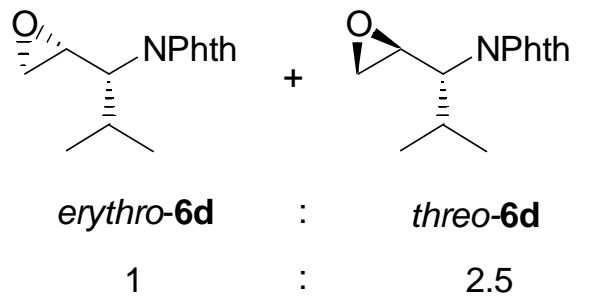

Pale yellow oil; analytical TLC (silica gel 60) (20\% EA in hexane), erythro-6d $\mathbf{R}_{f}=0.3$, threo-6d $\mathrm{R}_{f}=0.3 ;{ }^{1} \mathrm{H}$ NMR $\left(300 \mathrm{MHz}, \mathrm{CDCl}_{3}\right)$ ä 7.87-7.82 (m, 2H), 7.75-7.71 (m, 2H), 3.70-3.66 (m, 1H), 3.44-3.36 (m, 1H), $2.99(\mathrm{dd}, J=5.1,3.9 \mathrm{~Hz}$, threo- $2.5 / 3.5 \times 1 \mathrm{H})$, $2.75(\mathrm{dd}, J=5.0,2.5$, threo- $2.5 / 3.5 \times 1 \mathrm{H}), 2.69-2.61(\mathrm{~m}$, erythro- $1 / 3.5 \times 2 \mathrm{H}), 2.47(\mathrm{dd}$, $J=5.0,2.6 \mathrm{~Hz}$, erythro- $1 / 3.5 \times 1 \mathrm{H}), 1.19(\mathrm{~d}, J=6.7 \mathrm{~Hz}$, erythro- $1 / 3.5 \times 3 \mathrm{H}), 1.10(\mathrm{~d}, J$ $=6.7 \mathrm{~Hz}$, threo- $2.5 / 3.5 \times 3 \mathrm{H}), 0.89(\mathrm{~d}, J=6.7 \mathrm{~Hz}$, threo- $2.5 / 3.5 \times 3 \mathrm{H}), 0.87(\mathrm{~d}, J=6.7$ $\mathrm{Hz}$, erythro- $1 / 3.5$ x 3H); ${ }^{13} \mathrm{C}$ NMR $\left(75.47 \mathrm{MHz}, \mathrm{CDCl}_{3}\right)$ ä 168.3, 168.1, 134.1, 133.9, 131.7, 131.6, 123.3, 123.2, 61.4, 61.0, 51.9, 51.0, 48.9, 44.6, 30.0, 28.4, 19.9, 19.7, 19.5; IR (KBr) 1777, $1711 \mathrm{~cm}^{-1}$; EIMS $\mathrm{m} / z 245\left(\mathrm{M}^{+}\right)$; HRMS (EI) for $\mathrm{C}_{14} \mathrm{H}_{15} \mathrm{O}_{3} \mathrm{~N}$, calcd 245.1051, found 245.1047. 
${ }^{1}$ H NMR Spectra of Diastereomeric Mixtures of Epoxides 4

Table 1 , entry 1

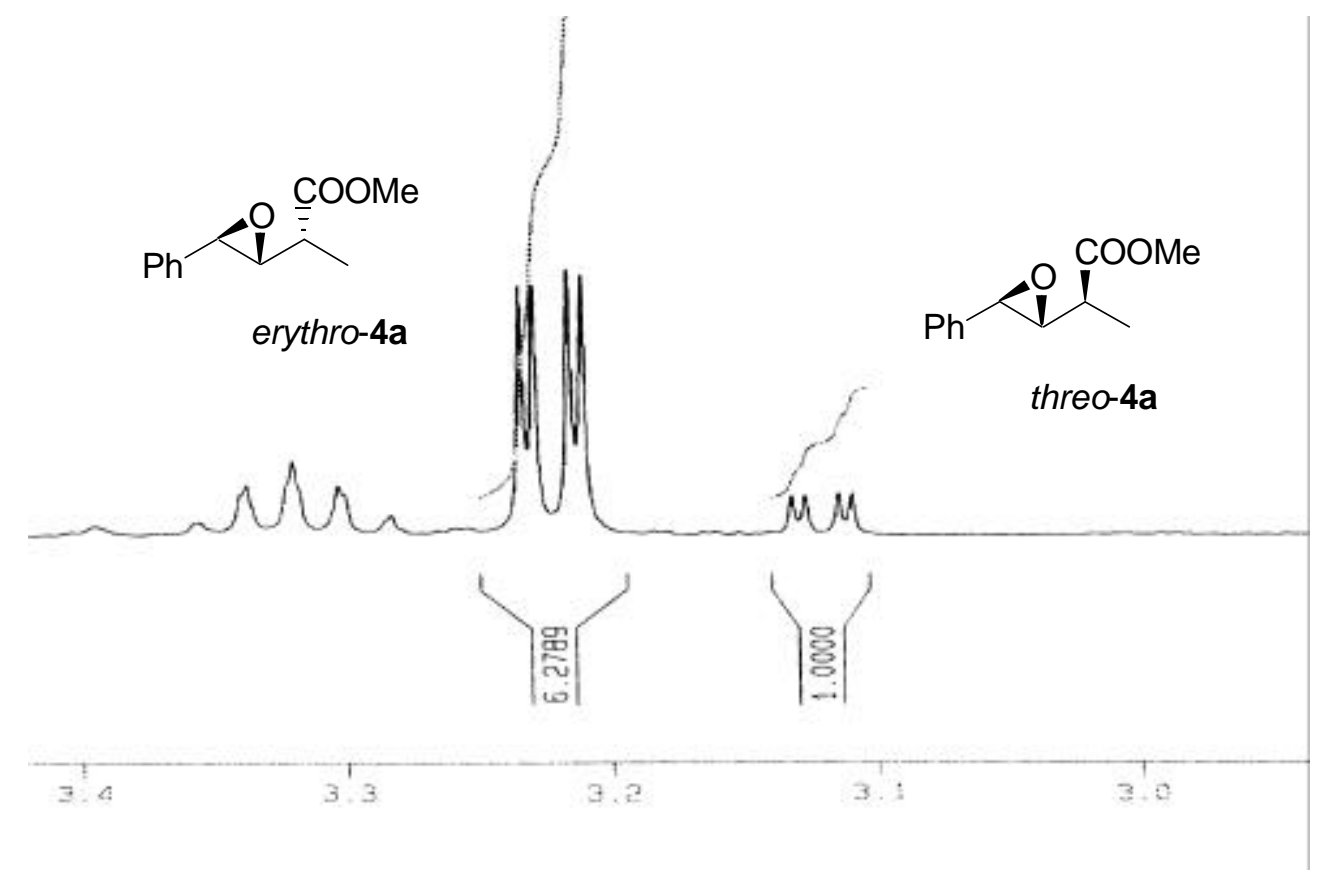

Table 1 , entry 6

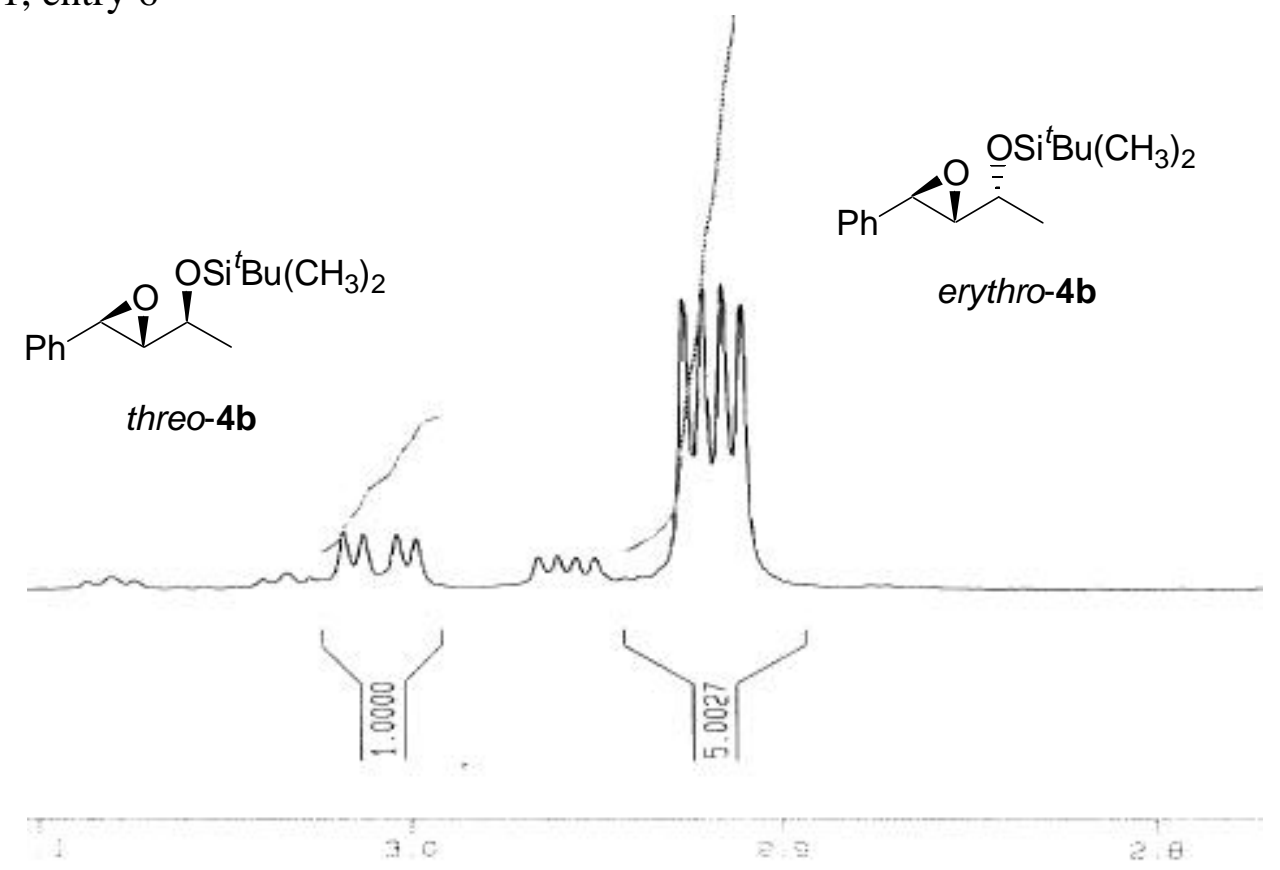


Table 3 , entry 2

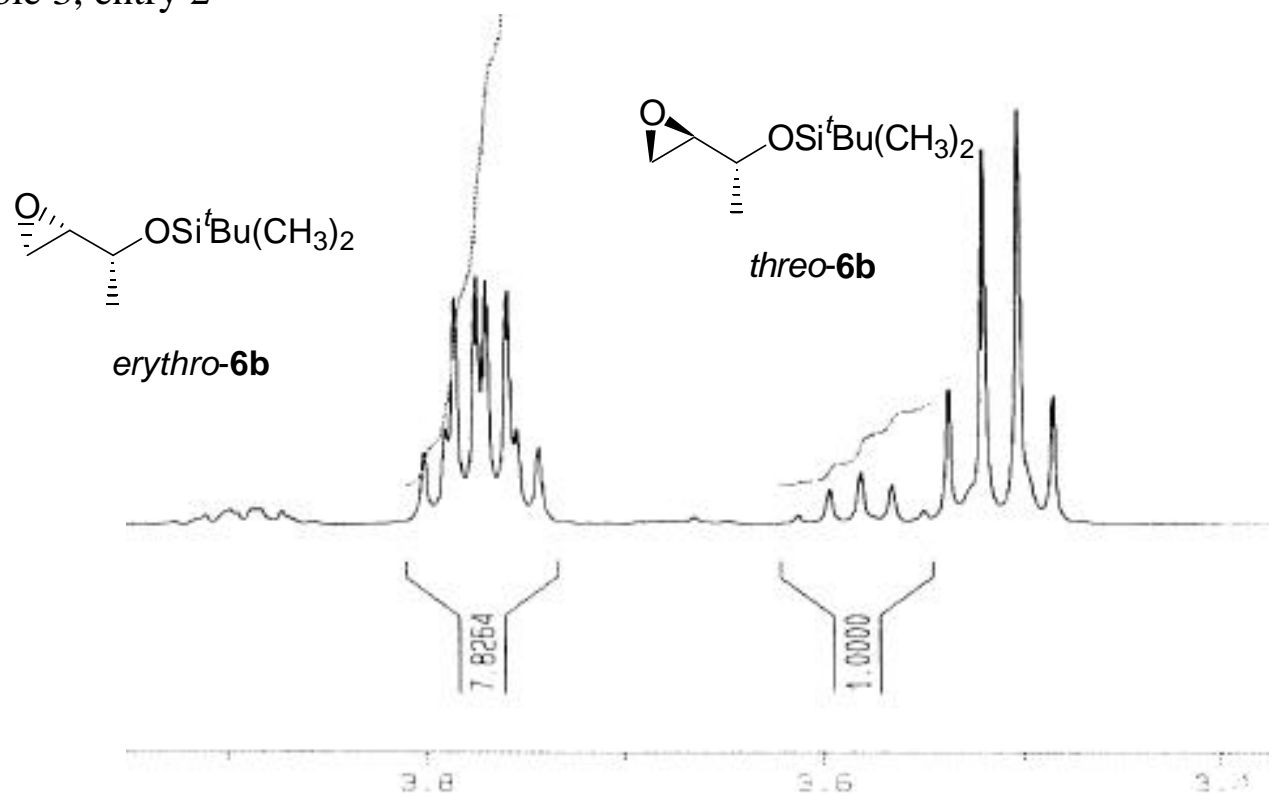

Table 3, entry 4

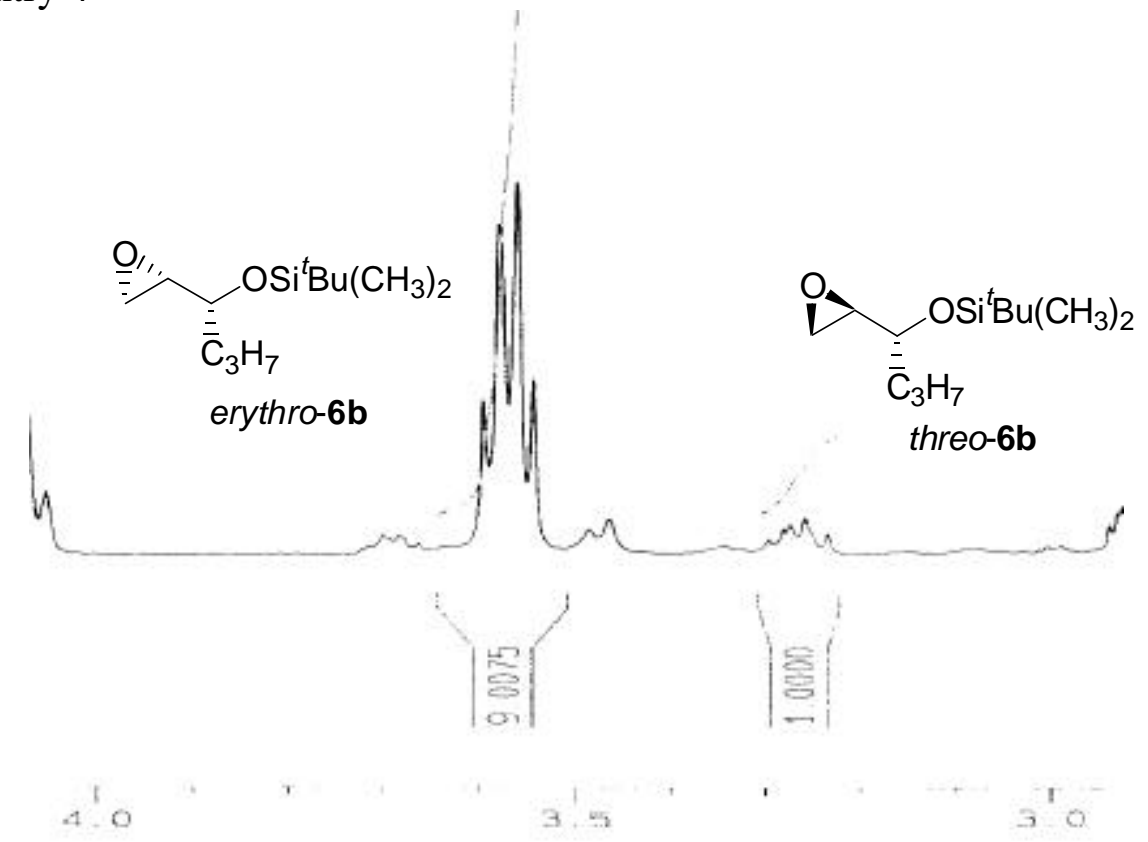


Table 3, entry 5

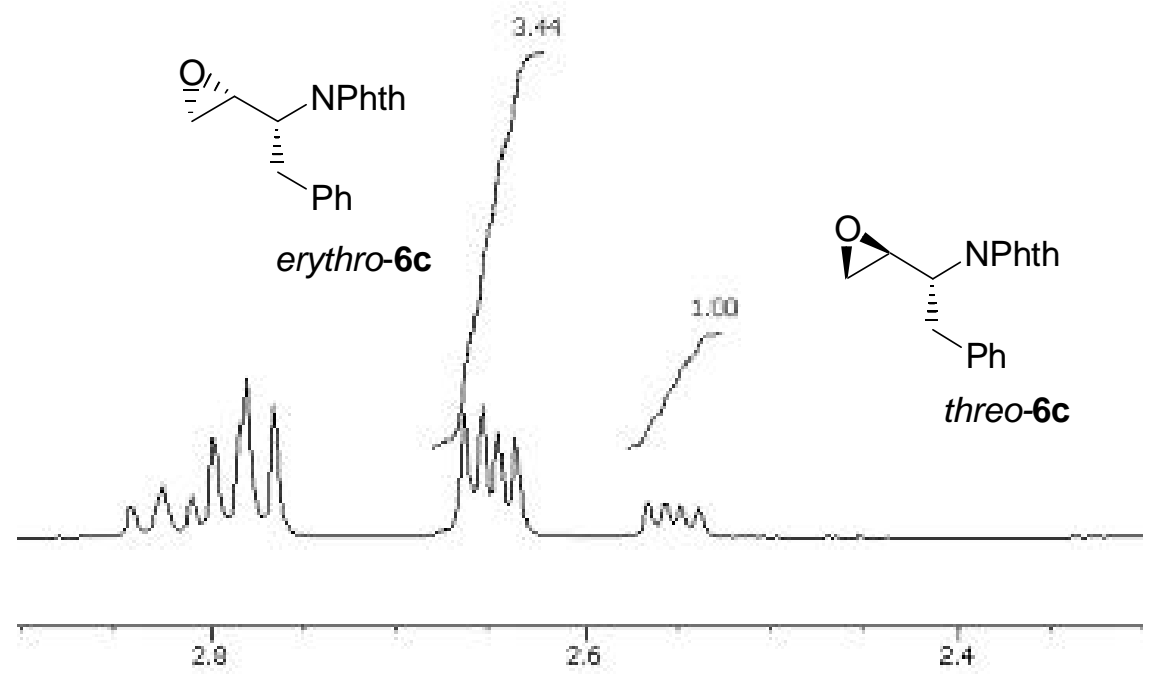

Table 3 , entry 8

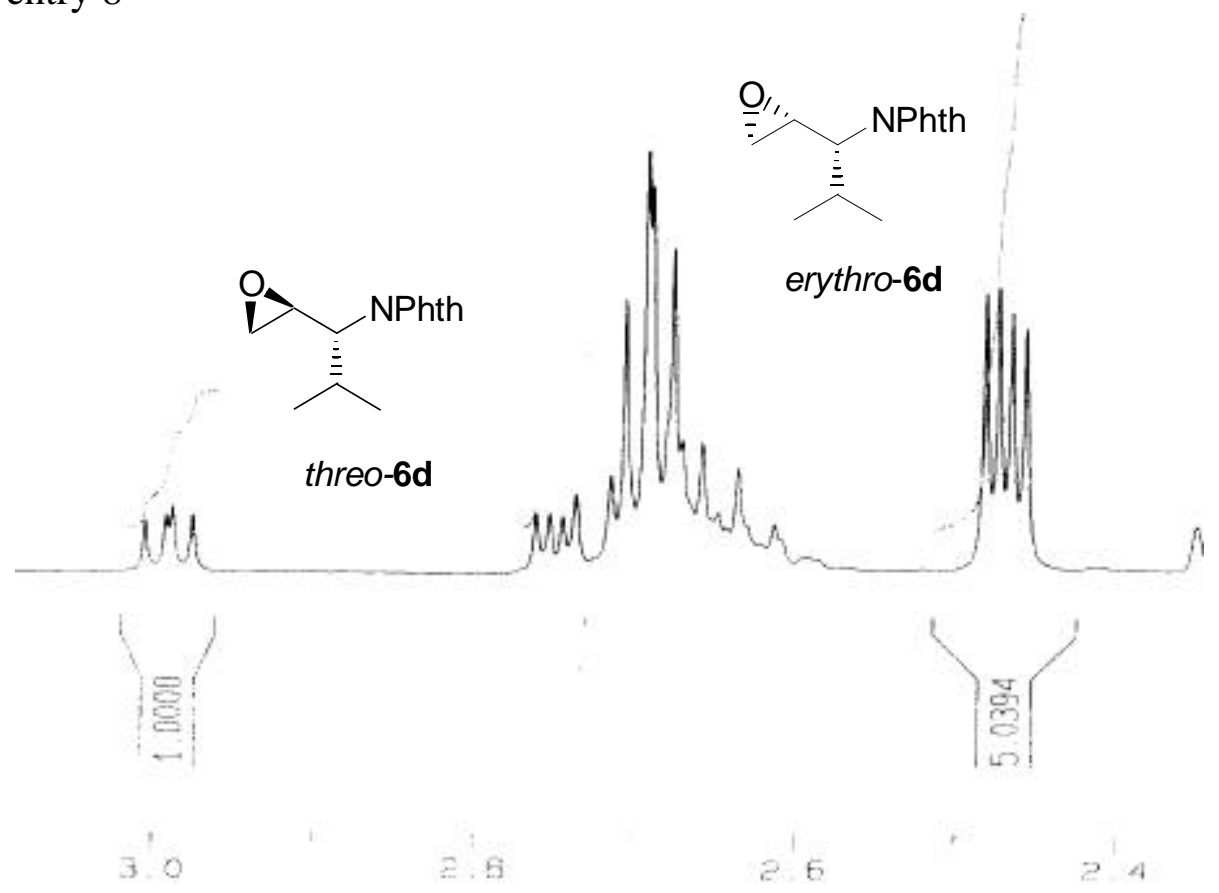


Determination of the $\alpha-8 / \beta-8$ Ratio with ${ }^{1} \mathrm{H}$ NMR

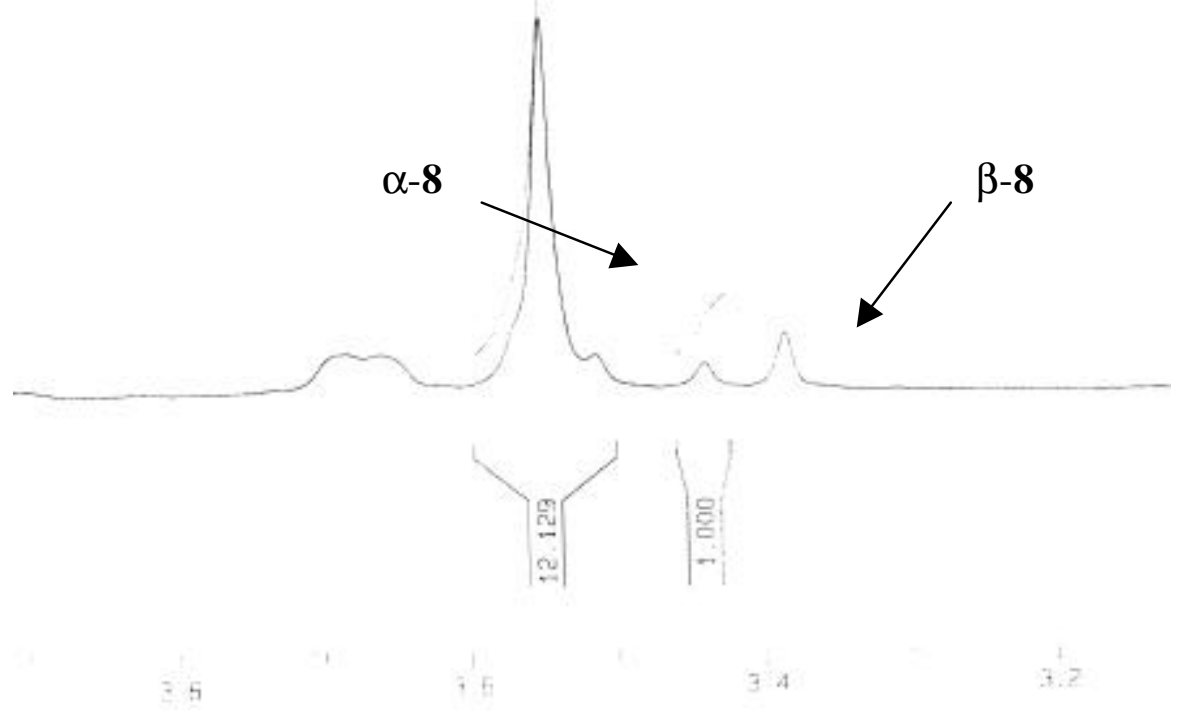




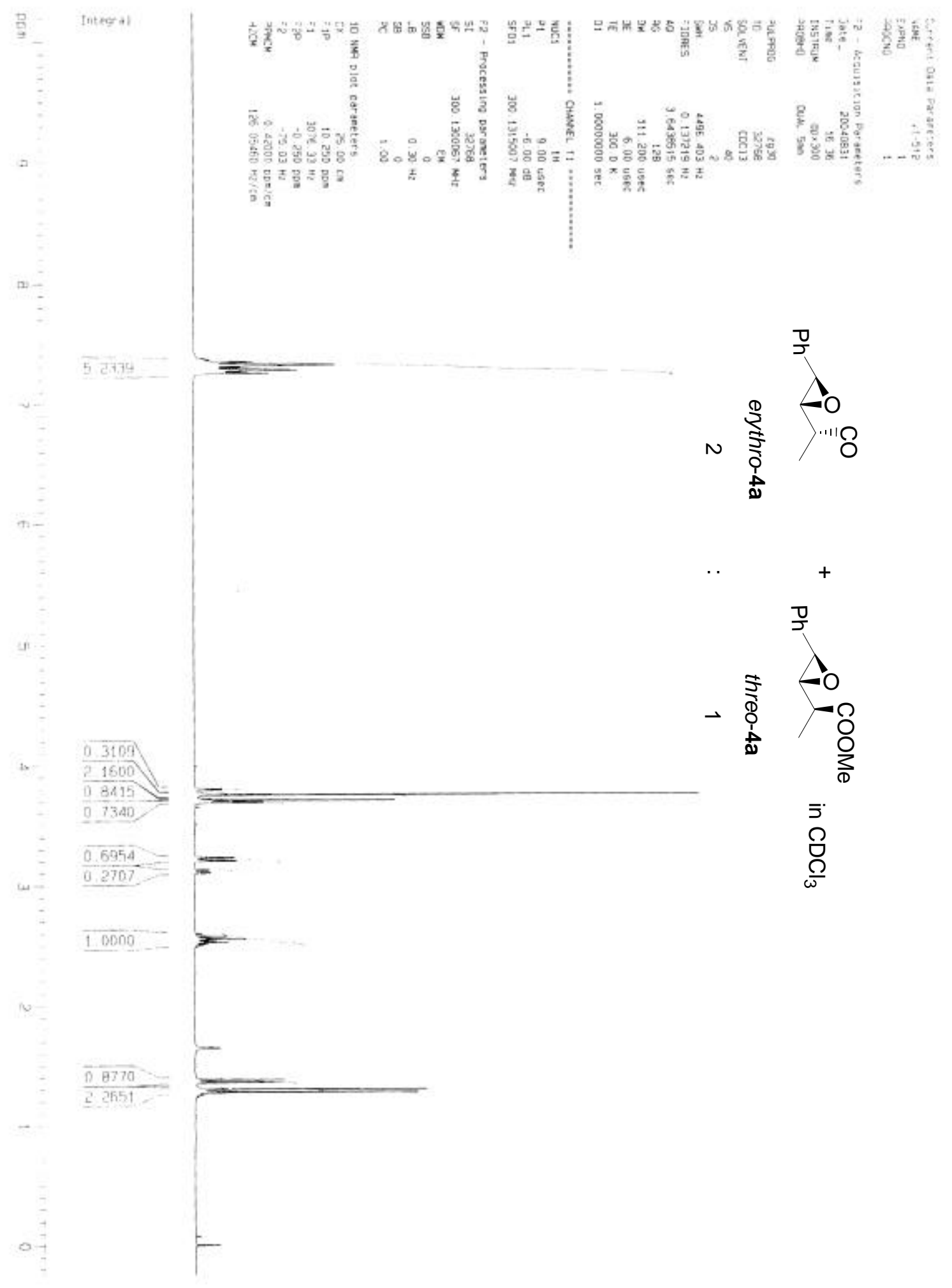




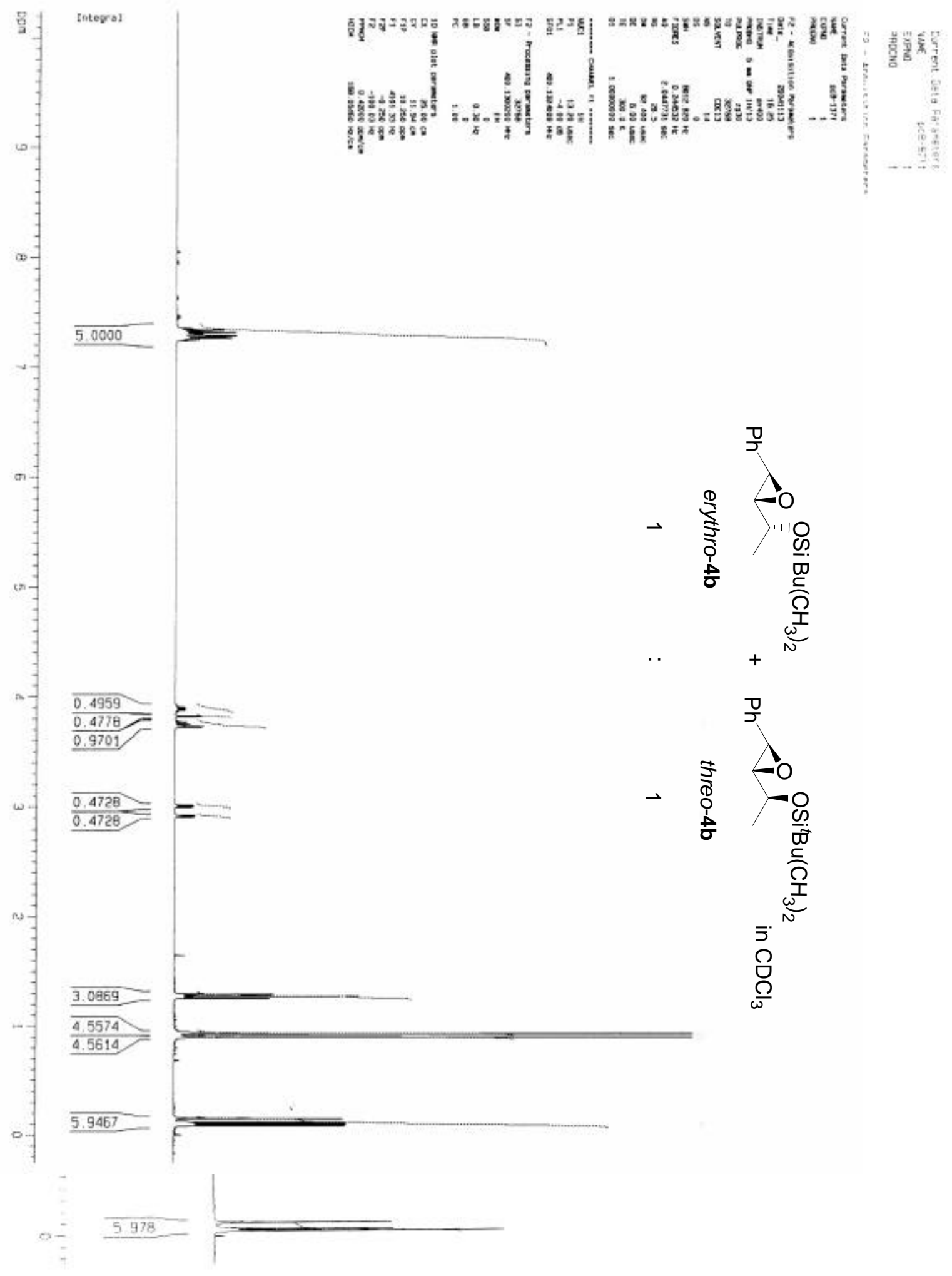




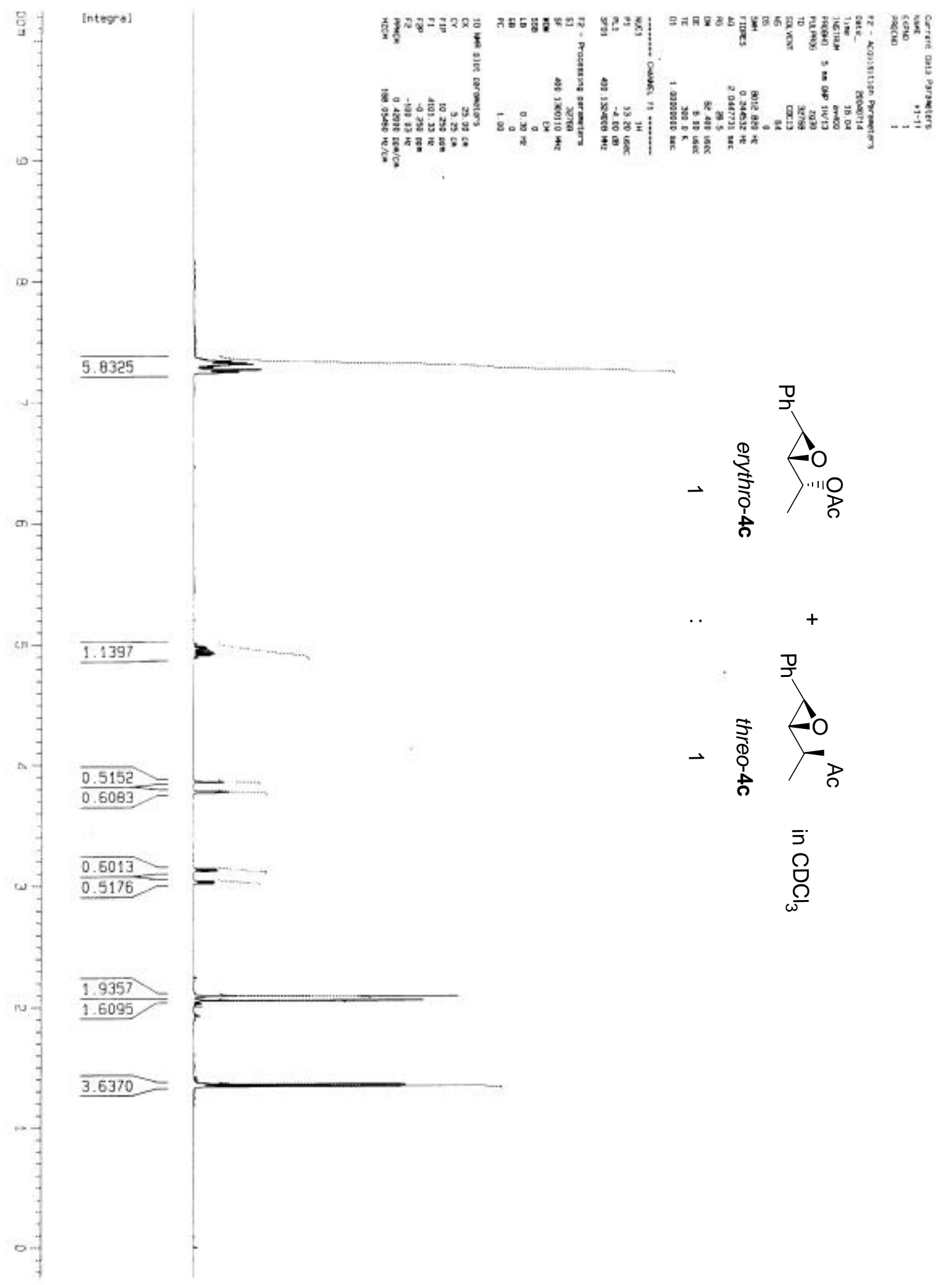




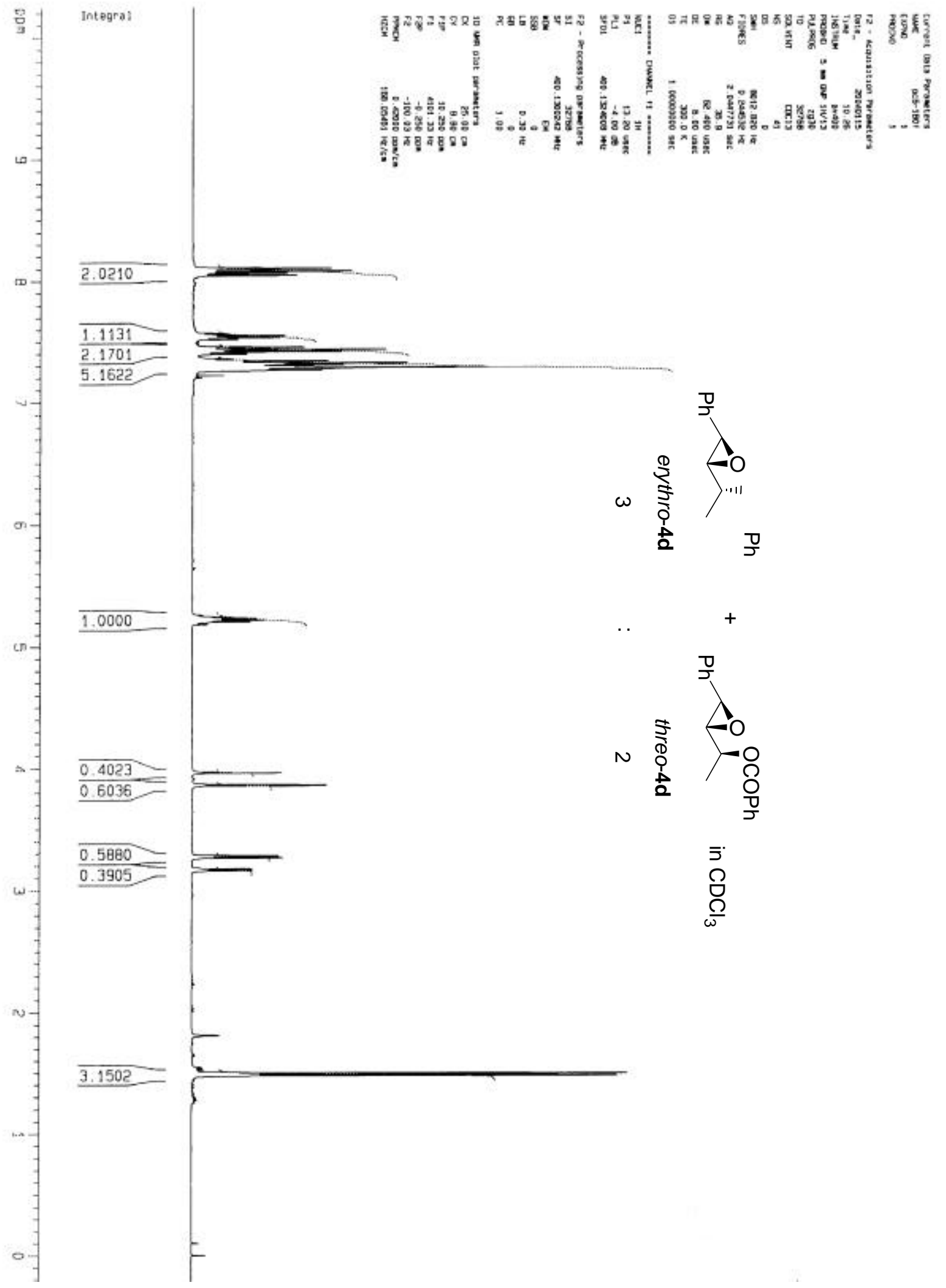




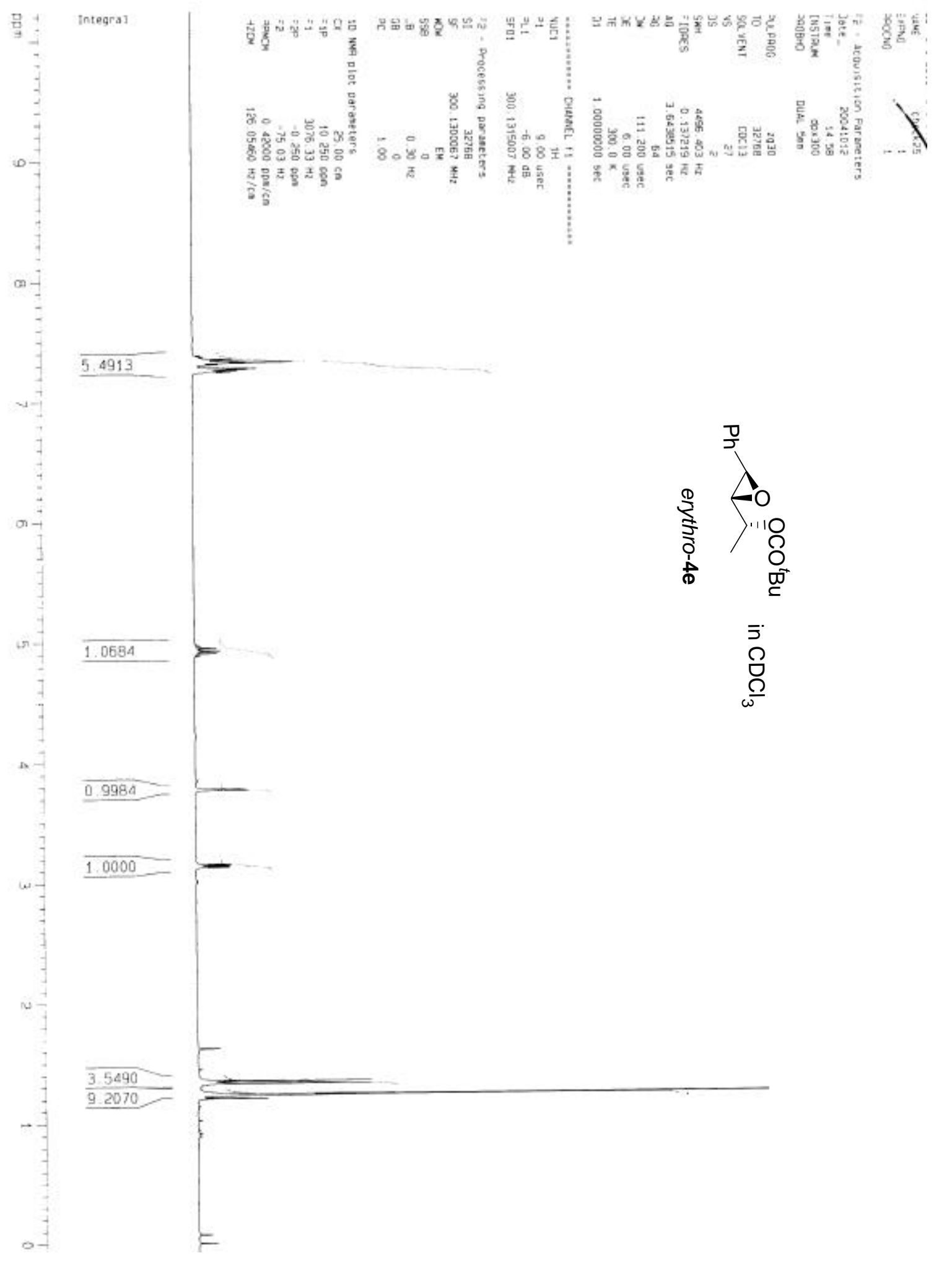




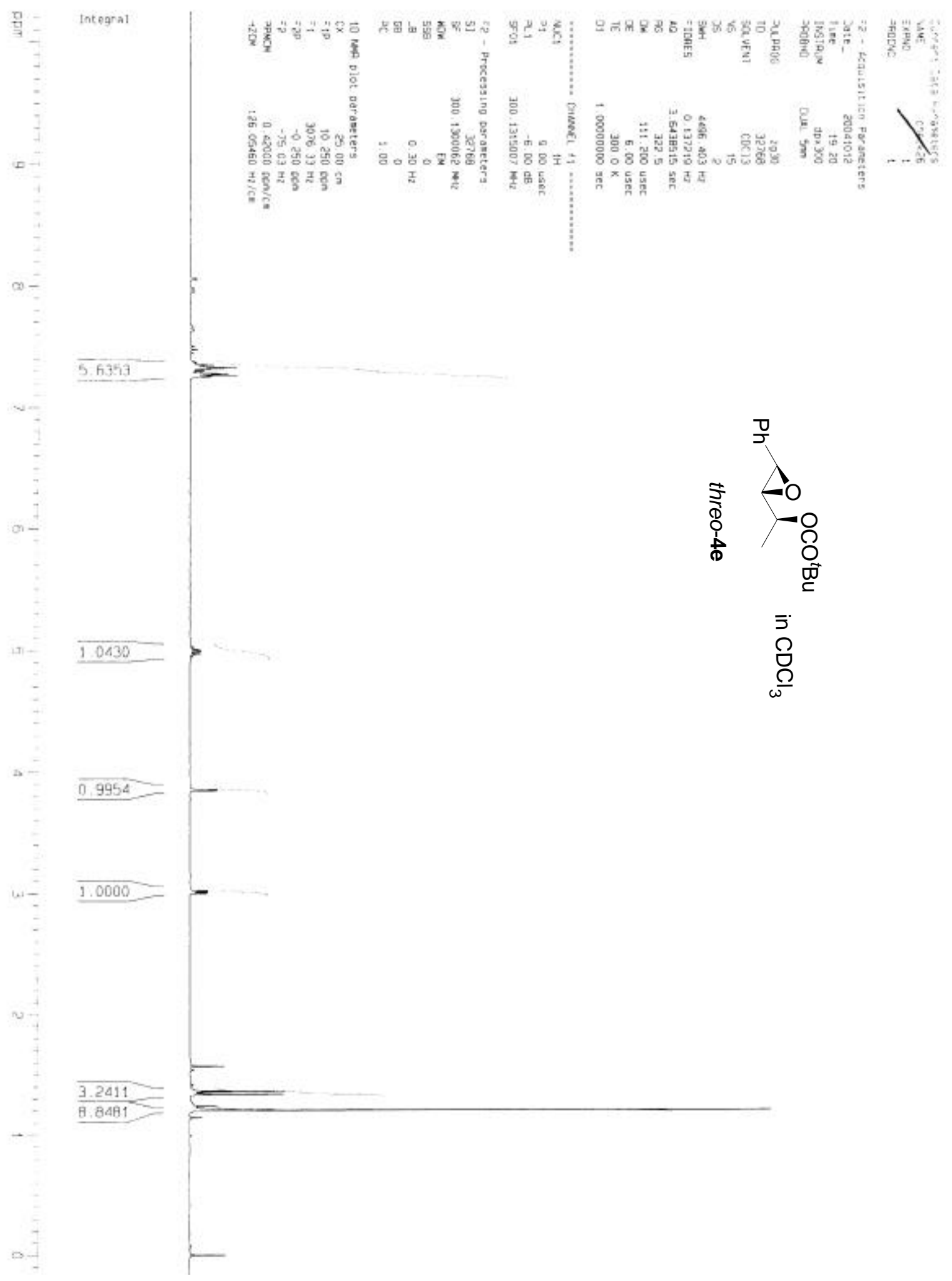




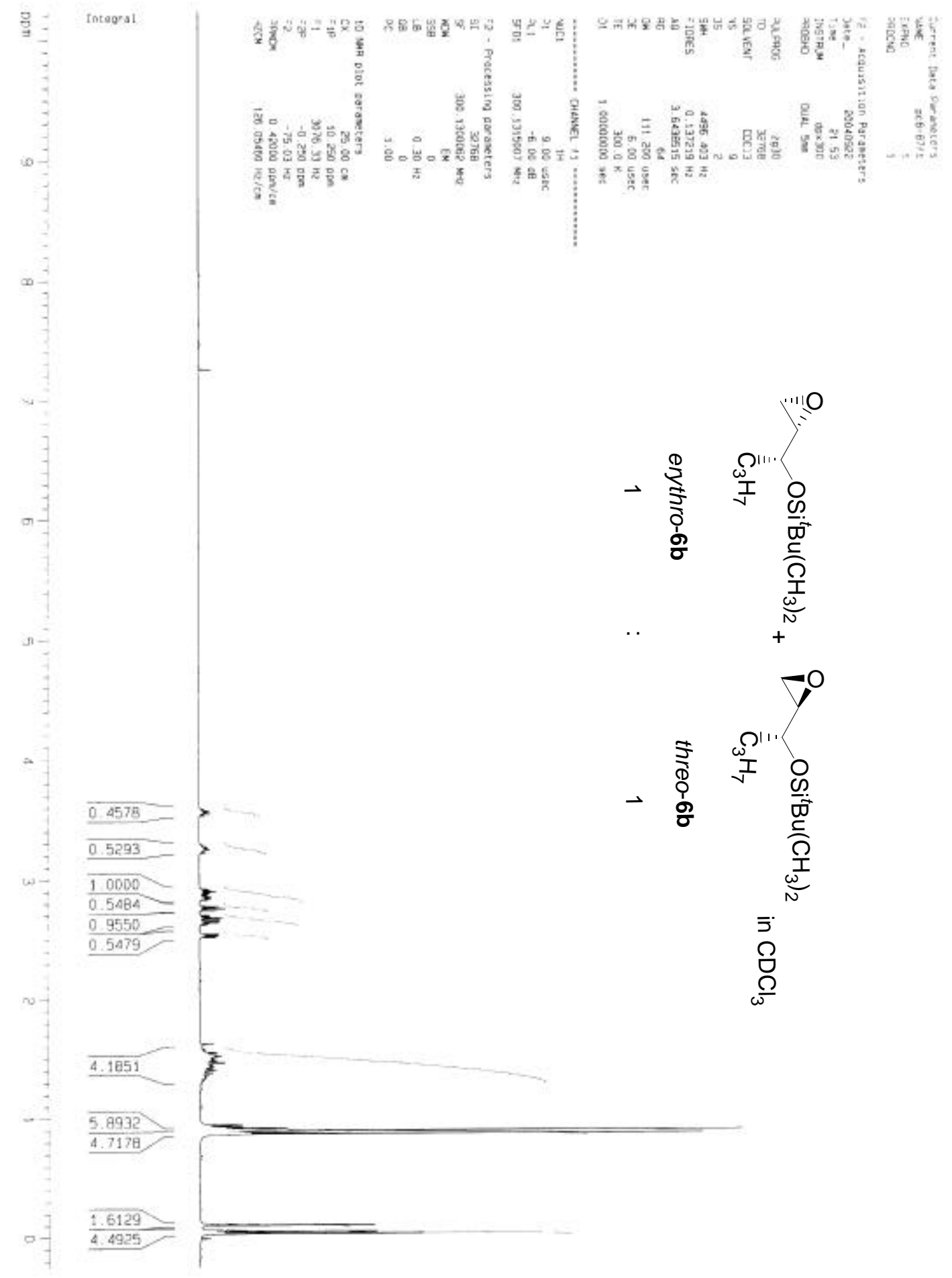




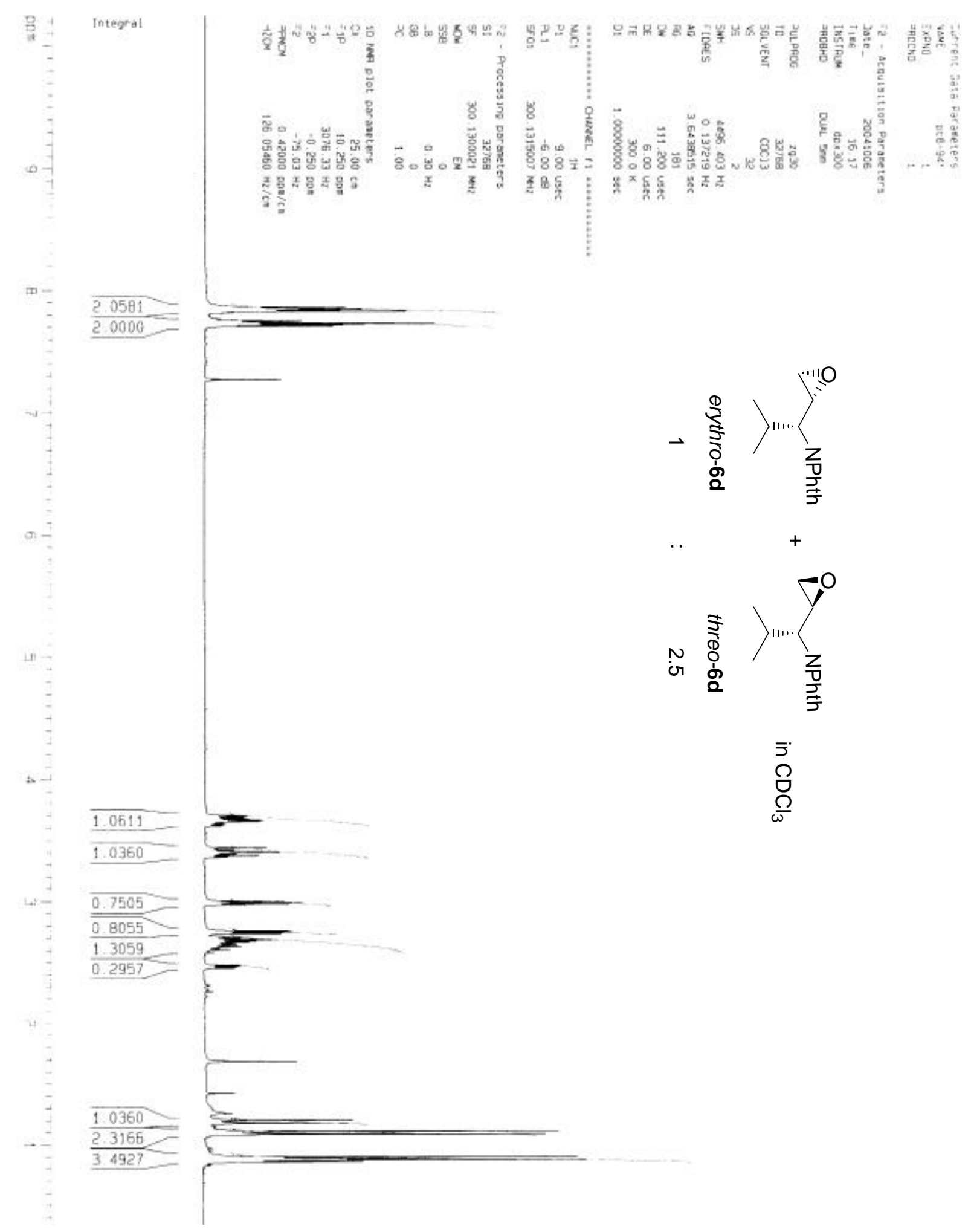




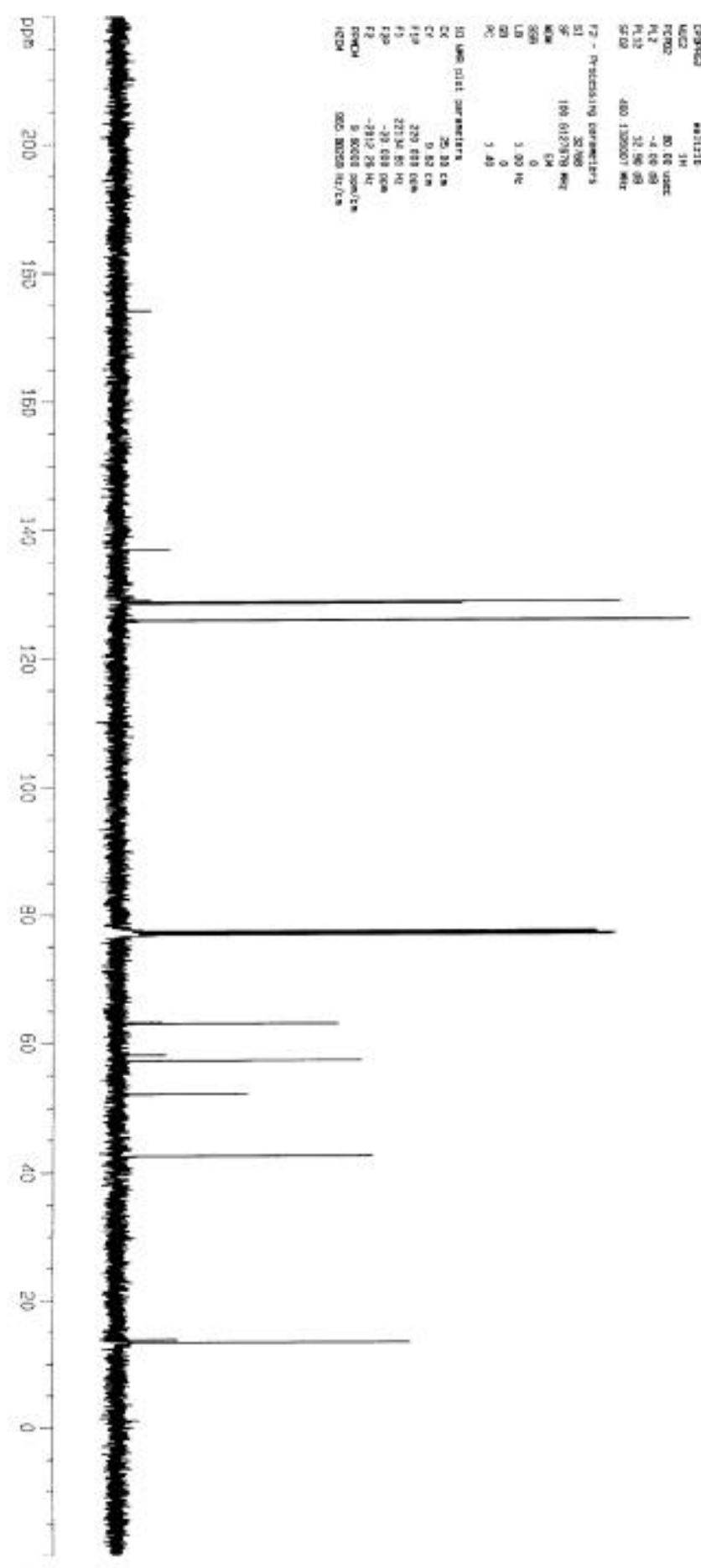

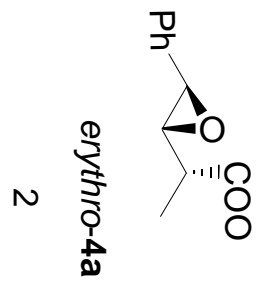

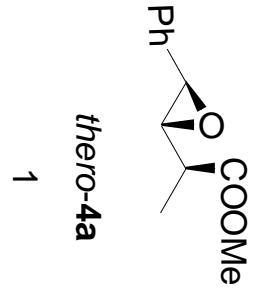

$$
\begin{aligned}
& \begin{array}{l}
\overline{5} \\
\stackrel{8}{\circ} \\
\stackrel{\Omega}{\omega}
\end{array}
\end{aligned}
$$




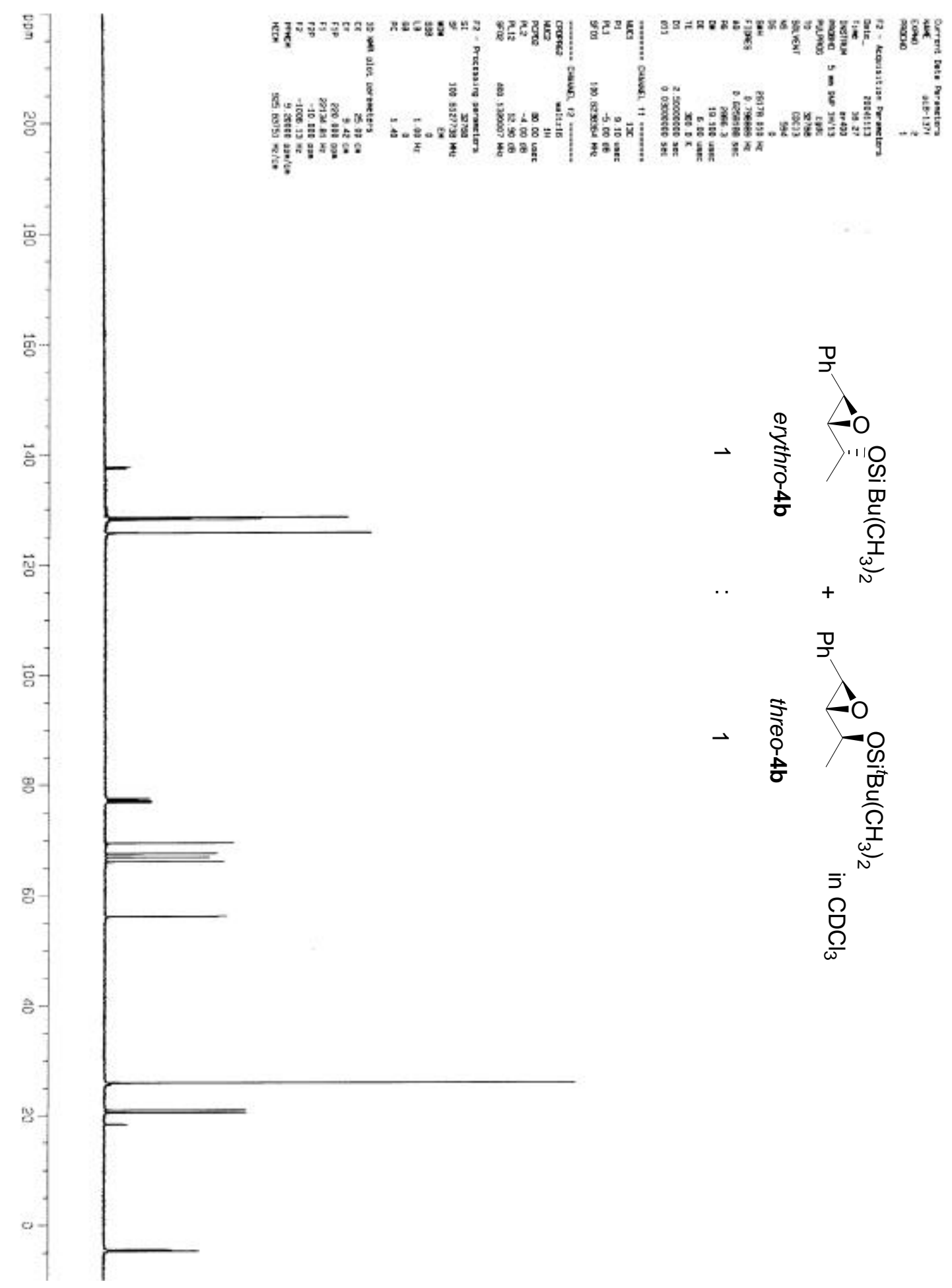




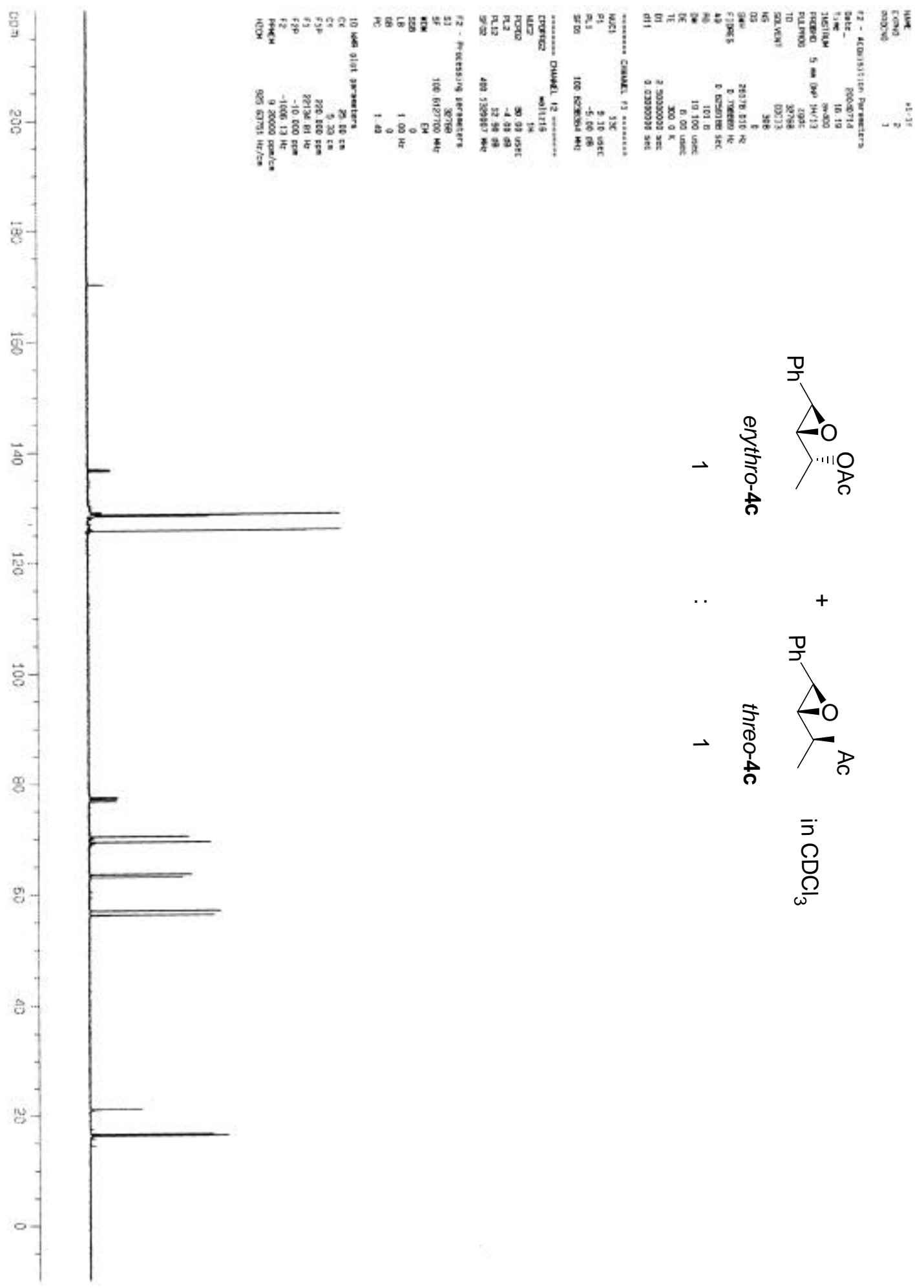




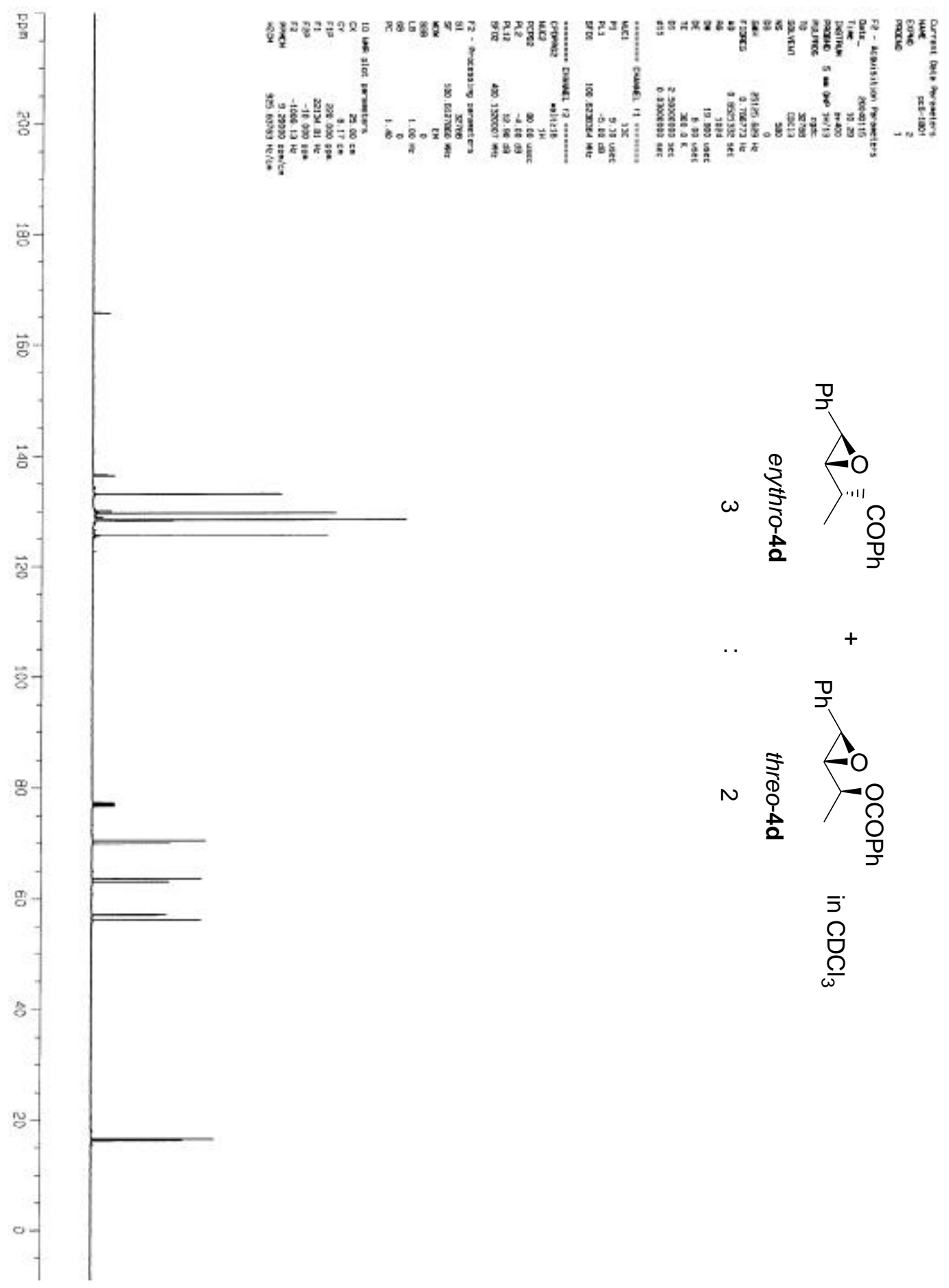




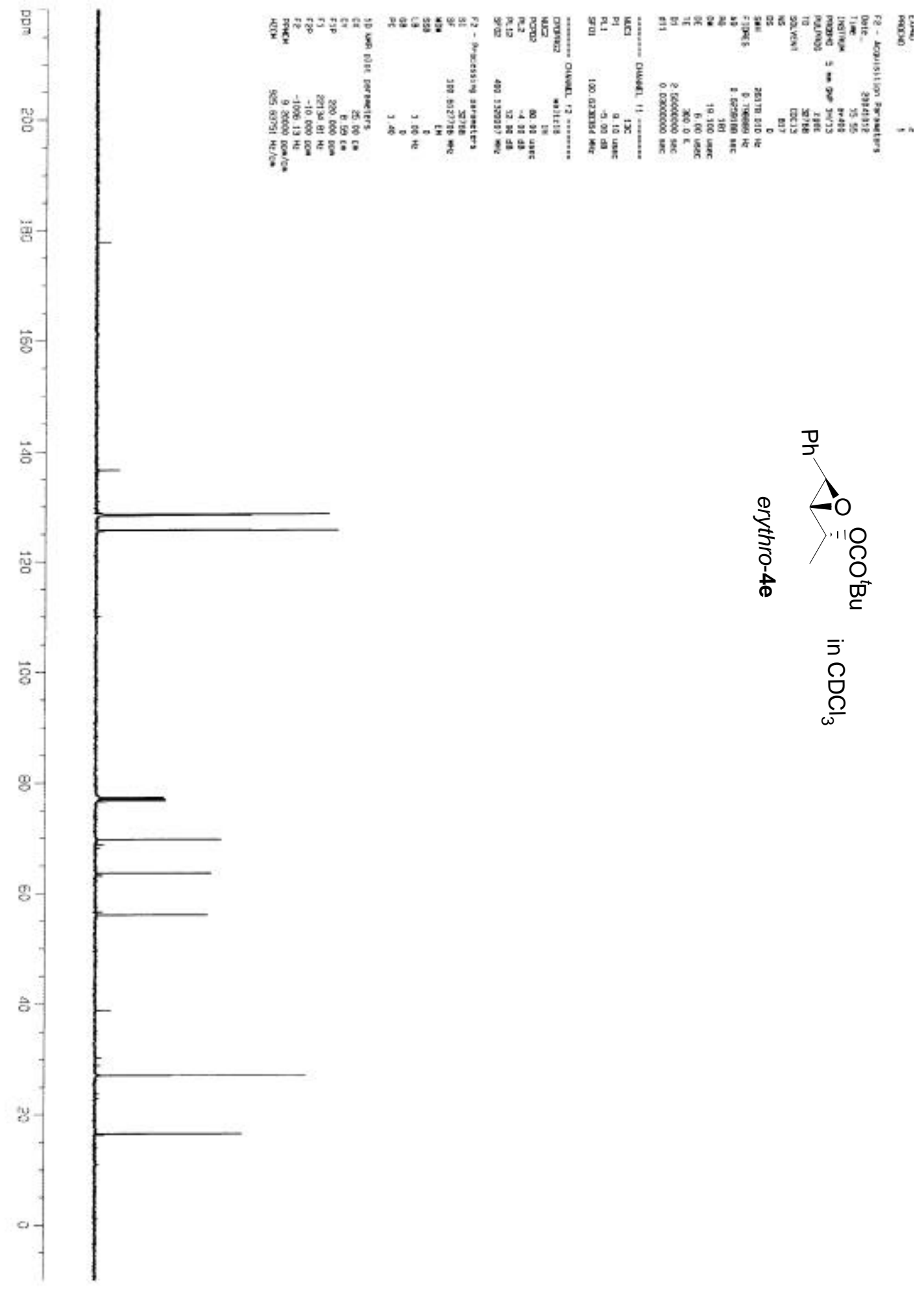




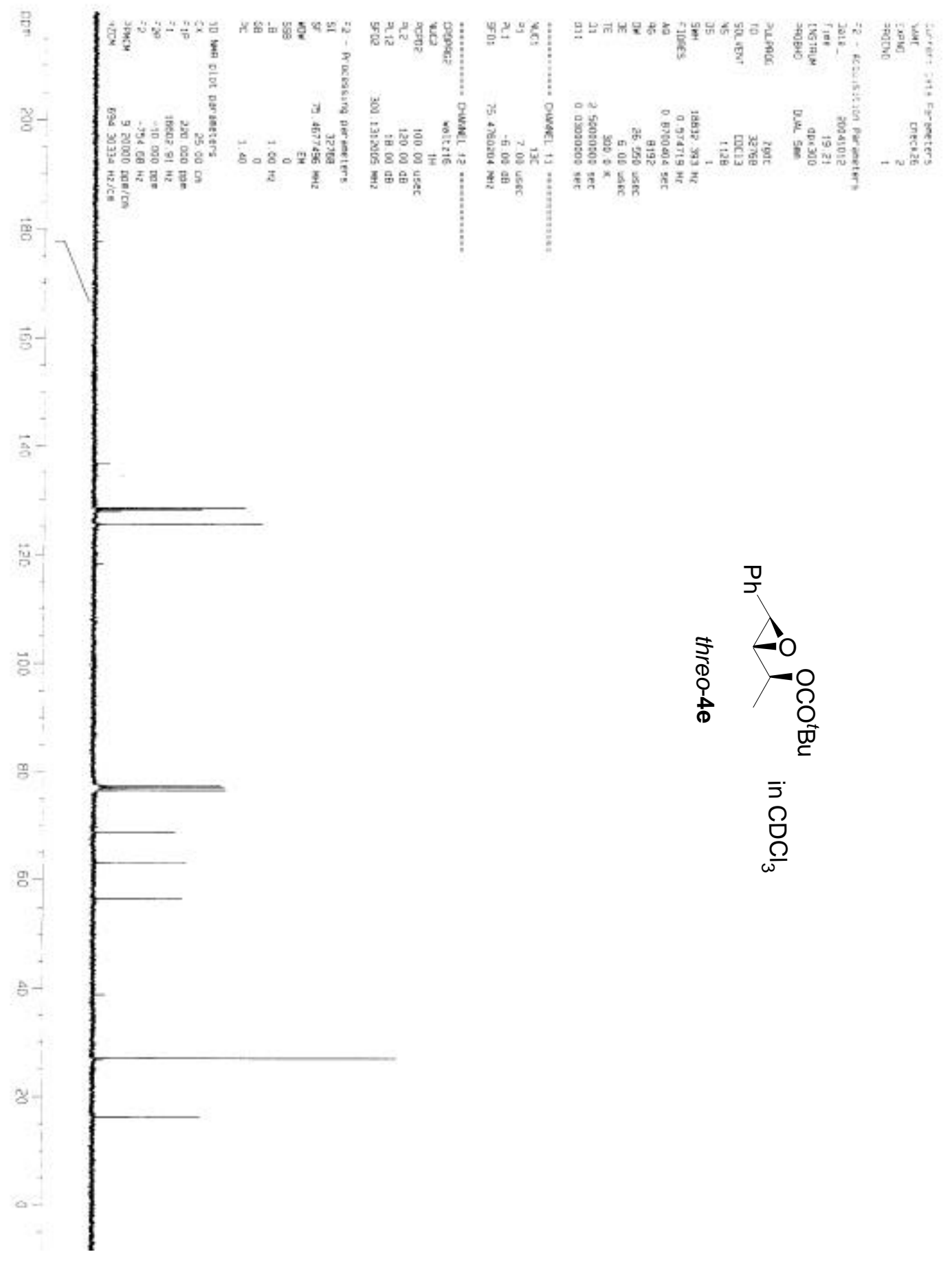




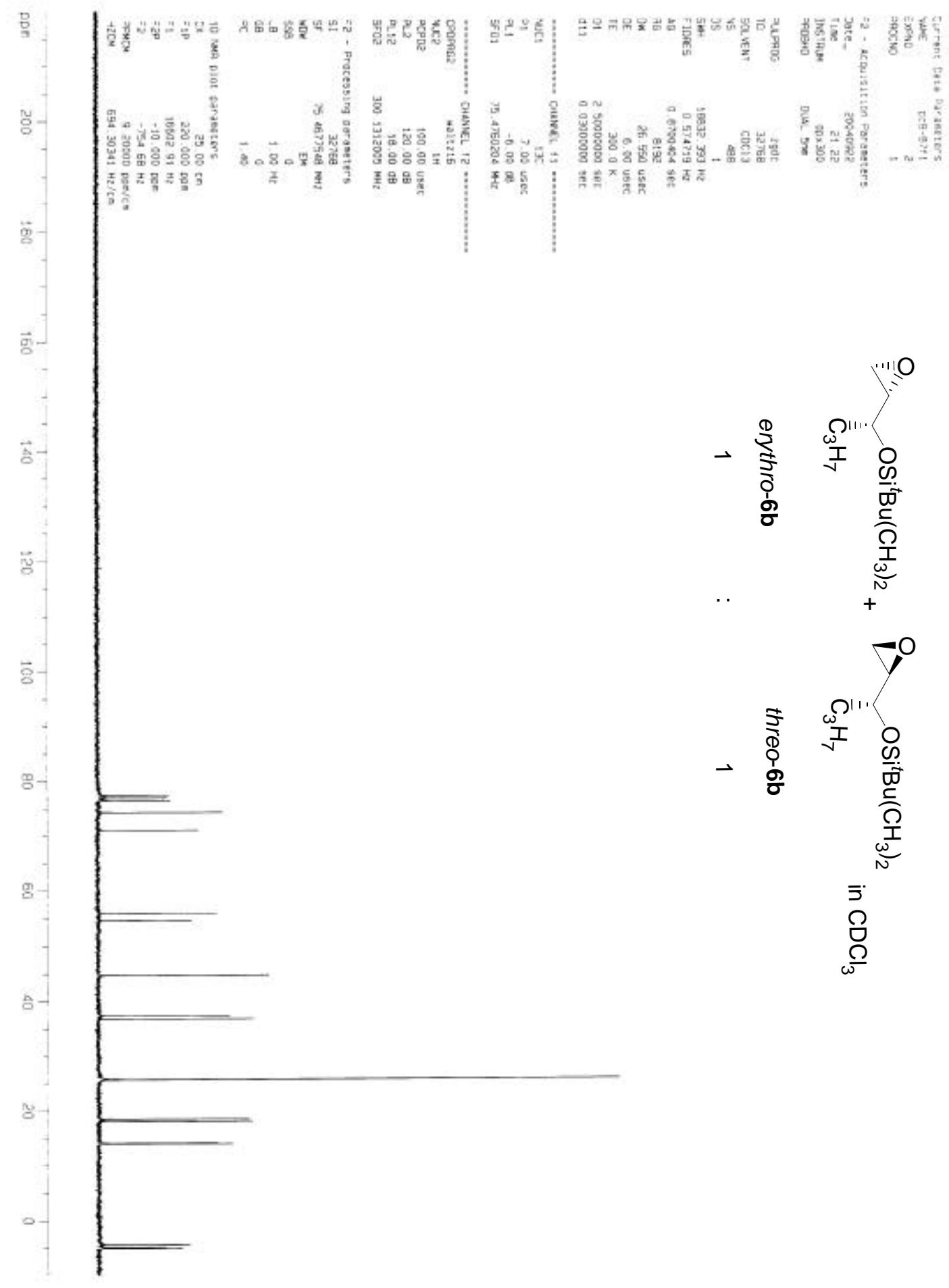




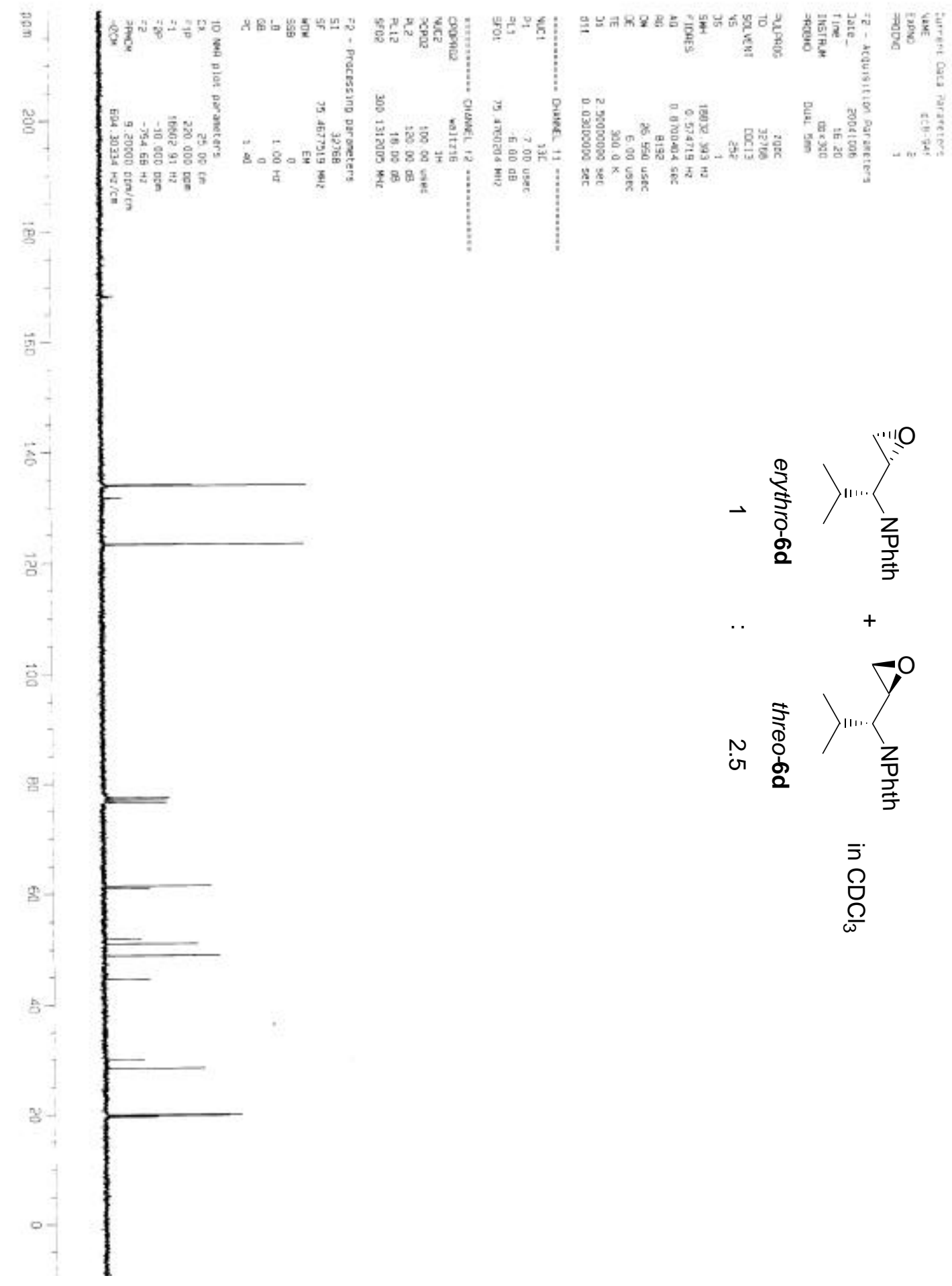

